\title{
HARMONIC ANALYSIS FOR GROUPS ACTING ON TRIANGLE BUILDINGS
}

\section{DONALD I. CARTWRIGHT and WOJCIECH MLOTKOWSKI}

(Received 18 March 1992; revised 2 June 1992)

Communicated by

\begin{abstract}
Let $\Delta$ be a thick building of type $\tilde{A}_{2}$, and let $\mathscr{V}$ be its set of vertices. We study a commutative algebra $\not$ of 'averaging' operators acting on the space of complex valued functions on $\mathscr{V}$. This algebra may be identified with a space of 'biradial functions' on $\mathcal{V}$, or with a convolution algebra of bi- $K$-invariant functions on $G$, if $G$ is a sufficiently large group of 'type-rotating' automorphisms of $\Delta$, and $K$ is the subgroup of $G$ fixing a given vertex. We describe the multiplicative functionals on $\mathscr{A}$ and the corresponding spherical functions. We consider the $C^{*}$-algebra induced by $\mathscr{A}$ on $\ell^{2}(\mathscr{V})$, find its spectrum $\Sigma$, prove positive definiteness of a kernel $k_{z}$ for each $z \in \Sigma$, find explicitly the spherical Plancherel formula for any group $G$ of type rotating automorphisms, and discuss the irreducibility of the unitary representations appearing therein. For the class of buildings $\Delta_{\mathscr{T}}$ arising from the groups $\Gamma_{\mathscr{T}}$ introduced in [2], this involves proving that the weak closure of $\mathscr{A}$ is maximal abelian in the von Neumann algebra generated by the left regular representation of $\Gamma \mathscr{g}$.
\end{abstract}

1991 Mathematics subject classification (Amer. Math. Soc.): primary: 51 E 24, 22 E 50; secondary: 43 A 35, 43 A 90.

Keywords and phrases: triangle buildings, spherical Plancherel formula.

\section{Introduction and notation}

Over the past ten years or more, there has been a great deal of effort devoted to the study of groups acting on a homogeneous tree $T$ of degree $q+1 \geq 3$,

(C) 1994 Australian Mathematical Society 0263-6115/94 \$A2.00+0.00

Research carried out while the second author was an ARC Research Associate at the University of New South Wales. 
and in particular to groups $\Gamma$ which act simply transitively on the vertices of $T$. The possible groups $\Gamma$ are well known (see [5, Chapter I, Theorem 6.3]). When $q$ is a prime power, $T$ is the (thick, type $\left.\tilde{A}_{1}\right)$ Tits building of $S L(2, F)$ for any local field $F$ whose residual field has order $q$ (see [11]). Thus $P G L(2, F)$ can be embedded in $\operatorname{Aut}(T)$, and all the possible groups $\Gamma$ can be realized as lattice subgroups of $P G L(2, F)$ (see [5, Appendix, Proposition 5.5]). Thus much of the theory of spherical representations of $\Gamma$ developed by intrinsic methods in, for example, [6] can be derived from the corresponding theory for $\operatorname{PGL}(2, F)$, especially in view of the recent theorem of Cowling and Steger [4] referred to below.

Now consider a (thick) Tits building $\Delta$ of type $\tilde{A}_{2}$, and denote by $\mathscr{V}$ its set of vertices. Following Tits [12], we shall call $\Delta$ a triangle building. The aim of the present paper is to study explicitly the 'spherical harmonic analysis' of groups acting on $\Delta$. In two recent papers, $[2,3]$, the groups $\Gamma$ were described which act simply transitively on $\mathscr{V}$. We shall be particularly interested in the harmonic analysis of these groups. Some, but not all, such $\Gamma$ can be embedded in $P G L(3, F)$ for some $F$.

The reader is referred to $[1,10]$ for the formal definition of a building and of a triangle building in particular. Only in Section 1 and Lemma 2.1 is any building theory used. Let us describe the features of these objects which we need here.

Firstly, a triangle building $\Delta$ is a simplicial complex consisting of vertices, edges and triangles. The triangles are also called chambers. Any two triangles can be joined by a 'gallery' of triangles so that two successive triangles have a common edge. In particular, (the vertices and edges of) $\Delta$ form a connected graph, which we always assume is locally finite, and we denote by $d(u, v)$ the usual graph-theoretic distance between vertices $u$ and $v$. Each vertex $v$ has a 'type' $\tau(v)=0,1$ or 2 , say, and each triangle has one vertex of each type. As $\Delta$ is thick, that is, as each edge lies on at least 3 triangles, one can show that each edge lies on the same finite number of triangles, and this number is denoted $q+1$. We call $q$ the order of $\Delta$. Unlike the case of trees, $\Delta$ is not determined by $q$ (see, for example, [12]).

If we fix a vertex $v_{0}$ of type 0 , say, the vertices $v$ satisfying $d\left(v_{0}, v\right)=1$, that is, the neighbours of $v_{0}$, have the structure of a finite projective plane: one lets $P$ and $L$ be the sets of neighbours of $v_{0}$ of types 1 and 2, respectively (or vice versa) and we call $u \in P$ and $v \in L$ incident if $u, v$ and $v_{0}$ lie on a common triangle. One has $|P|=|L|=q^{2}+q+1$.

An automorphism of $\Delta$ will be thought of as a bijection $g$ of $\mathscr{V}$ mapping edges to edges and chambers to chambers. An automorphism induces a per- 
mutation $\pi=\pi_{g}$ of types such that if $u \in \mathscr{V}$ has type $i$, then $g u$ has type $\pi(i)$. An automorphism is called type rotating if there is a $c \in\{0,1,2\}$ such that $\pi(i)=i+c(\bmod 3)$ for each $i$. The type rotating automorphisms form a subgroup $\operatorname{Aut}_{\mathrm{tr}}(\Delta)$ of index at most 2 in the full automorphism group.

There are two natural averaging operators $A^{+}$and $A^{-}$defined on the space of complex valued functions on $\mathscr{V}$ :

$$
\left(A^{ \pm} f\right)(u)=\frac{1}{q^{2}+q+1} \sum_{\substack{v: d(u, v)=1 \\ r(v)=x(u) \pm \bmod 3}} f(v) \quad(u \in \mathscr{V}) .
$$

We shall show in Section 2 that $A^{+}$and $A^{-}$commute, and generate an algebra $\mathscr{A}$ whose structure depends only on $q$. This algebra is linearly spanned by other averaging operators $A_{m, n}$. To describe these, we need to discuss the apartments of $\Delta$. These apartments are certain subcomplexes of $\Delta$, each of which is isomorphic to a plane tessellated regularly by equilateral triangles. Any two chambers lie on a common apartment. If $u$ and $v$ are two vertices of $\Delta$, we can find an apartment $A$ containing a sector having $u$ as its vertex, and rays $r=\left(v_{0}=u, v_{1}, \ldots\right)$ and $r^{\prime}=\left(v_{0}^{\prime}=u, v_{1}^{\prime}, \ldots\right)$, so that $v$ is at distance $n$ from $r$ and distance $m$ from $r^{\prime}$ and so that $\tau\left(v_{i+1}\right)=\tau\left(v_{i}\right)+1(\bmod 3)$ and $\tau\left(v_{i+1}^{\prime}\right)=\tau\left(v_{i}^{\prime}\right)-1(\bmod 3)$ for each $i \geq 0($ see Figure 1$)$.
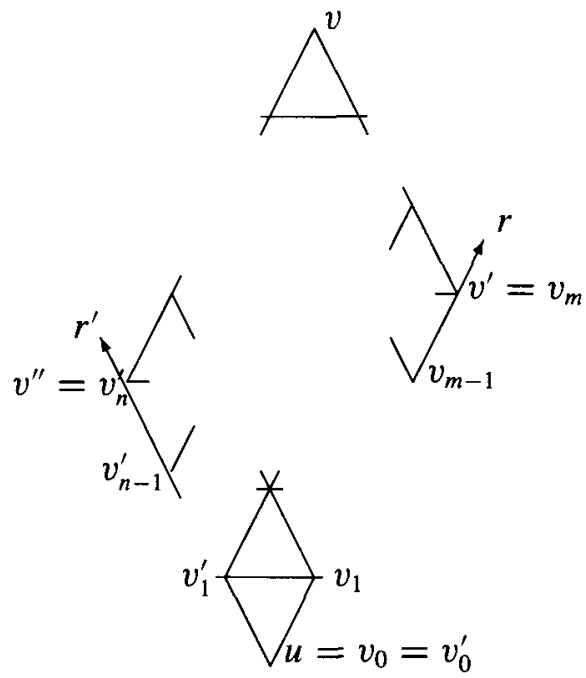

FIGURE 1 
In this case, we write $v \in S_{m, n}(u)$. The parallelogram $u v^{\prime} v v^{\prime \prime}$, which we call the convex hull of $u$ and $v$, is common to any apartment containing $u$ and $v[1$, p. 152], and so $S_{m, n}(u)$ is well defined. Any such subcomplex is contained in some apartment [1, p. 166]. Clearly $v \in S_{m, n}(u)$ if and only if $u \in S_{n, m}(v)$. We shall see that $N_{m, n}=\left|S_{m, n}(u)\right|$ does not depend on $u$. The operator $A_{m, n}$ is defined by

$$
\left(A_{m, n} f\right)(u)=\frac{1}{N_{m, n}} \sum_{y \in S_{m, n}(u)} f(y) \quad(u \in \mathscr{V}) .
$$

Thus $A^{+}=A_{1,0}$ and $A^{-}=A_{0,1}$.

Let $G$ be a group of type rotating automorphisms of $\Delta$, and let $\lambda$ denote the representation of $G$ induced on the space of complex valued functions on $\mathscr{V}$ : $(\lambda(g) f)(u)=f\left(g^{-1} u\right)$. The significance of the type rotating hypothesis is that it implies that each $\lambda(g)$ commutes with each $A \in \mathscr{A}$. This is because $g\left(S_{m, n}(u)\right)=S_{m, n}(g u)$.

We shall repeatedly refer below to two classes of triangle buildings:

(a) Let $F$ be local field whose residual field has order $q$. Then there is a triangle building $\Delta_{F}$ associated with $S L(3, F)$, (see [1, Section VI 9F] or [10, Section 9.2]). Briefly, it is constructed as follows. Let $v$ be the valuation on $F$, let $\mathscr{O}=\{x \in F: v(x) \geq 0\}$, and let $\varpi \in \mathscr{O}$ satisfy $v(\varpi)=1$. Let $L$ be a lattice in $V=F^{3}$, that is, an $\mathscr{O}$-submodule of $V$ of the form $\left\{a_{1} v_{1}+a_{2} v_{2}+a_{3} v_{3}: a_{1}, a_{2}, a_{3} \in \mathscr{O}\right\}$, where $\left\{v_{1}, v_{2}, v_{3}\right\}$ is a basis for $V$ over $F$. Lattices $L$ and $L^{\prime}$ are called equivalent if $L^{\prime}=t L$ for some $t \in F$. The vertices of $\Delta_{F}$ are the lattice classes $[L]$. The chambers of $\Delta_{F}$ consist of triples of distinct vertices [ $\left.L_{1}\right],\left[L_{2}\right]$ and $\left[L_{3}\right]$ such that $L_{1} \supset L_{2} \supset L_{3} \supset \varpi L_{1}$. If $\left\{e_{1}, e_{2}, e_{3}\right\}$ is the usual basis of $F^{3}$, let $L_{0}$ be the $\mathscr{O}$-submodule generated by $e_{1}, e_{2}$ and $e_{3}$. The group $\mathscr{G}=P G L(3, F)$ acts on $\Delta_{F}$ by left multiplication. The type of $\left[g L_{0}\right]$ is $v(\operatorname{det}(g))(\bmod 3)$ for each $g \in G L(3, F)$ and so $\mathscr{G} \subset \operatorname{Aut}_{\mathrm{rr}}\left(\Delta_{F}\right)$. The stabilizer of $\left[L_{0}\right]$ is the image $\mathscr{K}$ in $\mathscr{G}$ of $G L(3, \mathscr{O})$. One apartment $A_{0}$ in $\Delta_{F}$ consists of the subcomplex whose vertices are $\left[L_{l, m, n}\right]$, where $l, m, n \in \mathbb{Z}$ and $L_{l, m, n}$ is the $\mathscr{O}$-submodule generated by $\varpi^{l} e_{1}, \varpi^{m} e_{2}$ and $\varpi^{n} e_{3}$. The other apartments are $g A_{0}$, for $g \in \mathscr{G}$. If $u=\left[L_{0}\right]$, then $S_{m, n}(u)$ is the $\mathscr{K}$-orbit of $\left[L_{0, m, m+n}\right]$.

(b) Another class of triangle buildings was introduced in $[2,3]$. Let $(P, L)$ be a projective plane of order $q$, let $\lambda: P \rightarrow L$ be a bijection, and let $\mathscr{T}$ be a set of triples $(x, y, z)$, where $x, y, z \in P$, with the following properties:

(i) given $x, y \in P$, then $(x, y, z) \in \mathscr{T}$ for some $z \in P$ if and only if $y$ and $\lambda(x)$ are incident;

(ii) $(x, y, z) \in \mathscr{T}$ implies that $(y, z, x) \in \mathscr{T}$; 
(iii) given $x, y \in P$, then $(x, y, z) \in \mathscr{T}$ for at most one $z \in P$. Let $\left\{a_{x}: x \in P\right\}$ be $|P|$ distinct letters, and form the abstract group

$$
\left.\Gamma_{\mathscr{T}}=\left\langle\left\{a_{x}: x \in P\right\}\right| a_{x} a_{y} a_{z}=1 \text { for each }(x, y, z) \in \mathscr{T}\right\rangle
$$

Then by [2, Theorem 3.4] $\mathscr{T}$ gives rise to a triangle building $\Delta_{\mathscr{T}}$ whose vertices and edges form the Cayley graph of $\Gamma_{\mathscr{T}}$ with respect to the generators $a_{x}$ and their inverses, and whose chambers are the sets $\left\{g, g a_{x}^{-1}, g a_{y}\right\}$, where $g \in \Gamma_{\mathscr{T}}$, and $x, y \in P$ with $(x, y, z) \in \mathscr{T}$ for some $z \in P$. The type of $g \in \Gamma_{\mathscr{T}}$ is $\tau(g)$, where $\tau: \Gamma \rightarrow \mathbb{Z} / 3 \mathbb{Z}$ is the homomorphism determined by $\tau\left(a_{x}\right)=\overline{1}$ for each $x \in P$. Of course $\Gamma_{\mathscr{T}}$ acts simply transitively on the vertices of $\Delta_{\mathscr{T}}$, and does so in a type-rotating way. It was shown in [2] that any triangle building on whose vertices a group acts simply transitively and in a type rotating way is isomorphic to a building $\Delta_{\mathscr{T}}$. If $g \in \Gamma_{\mathscr{T}}$, any minimal word for $g$ in the generators $a_{x}$ and their inverses contains the same number of generators and the same number of inverse generators (see Section 6). If $u$ is the identity element 1 in $\Gamma_{\mathscr{T}}$, then $S_{m, n}(u)$ consists of elements $g \in \Gamma_{\mathscr{T}}$ for which these numbers are $m$ and $n$, respectively.

Let us summarize the contents of the paper. As mentioned above, the operators $A^{+}$and $A^{-}$generate a commutative algebra $\mathscr{A}$ whose structure depends only on $q$. This algebra may also be identified with a space of 'biradial functions'. In Section 3, we calculate explicitly the multiplicative functionals $h$ on $\mathscr{A}$. These may be indexed by pairs $(z, w)$ of complex numbers, so that $h_{z, w}$ is the unique multiplicative functional $h$ such that $h\left(A^{+}\right)=z$ and $h\left(A^{-}\right)=w$. They may also be naturally indexed by the group $S=\left\{s=\left(s_{1}, s_{2}, s_{3}\right) \in \mathbb{C}^{3}: s_{1} s_{2} s_{3}=1\right\}$, the two indexing methods being connected by

$$
z=\frac{q}{q^{2}+q+1}\left(s_{1}+s_{2}+s_{3}\right) \quad \text { and } \quad w=\frac{q}{q^{2}+q+1}\left(\frac{1}{s_{1}}+\frac{1}{s_{2}}+\frac{1}{s_{3}}\right) .
$$

When $\Delta=\Delta_{F}, \mathscr{A}$ is isomorphic to the algebra of bi- $\mathscr{K}$-invariant functions on $\mathscr{G}=P G L(3, F)$. The multiplicative functionals for the closely related algebra of bi- $K$-invariant functions on $S L(3, F)$, where $K=S L(3, \mathscr{O})$, may be found in the book [7] as a very special case. However, as $P G L$ is not simply connected, the present situation is not quite covered by that book. In any case, our methods are quite different from those in [7] and completely elementary.

In Section 4, we consider $\mathscr{A}$ as an algebra of operators on $\ell^{2}(\mathscr{V})$, and calculate the spectrum of the commutative $C^{*}$-algebra obtained by taking the closure of $\mathscr{A}$ with respect to the corresponding operator norm. It is a certain hypocycloid $\Sigma$ 
(see Figure 4). We also prove that the kernel corresponding to each $z \in \Sigma$ is positive definite.

In Section 5, we calculate the associated Plancherel measure $\mu$ on this hypocycloid. According to a general theorem, the left regular representation $\lambda$ of a closed subgroup $G$ of $\operatorname{Aut}_{\text {tr }}(\Delta)$ may be written as a direct integral over $\Sigma$, with respect to $\mu$, of unitary representations $\pi_{z}$ (see, for example, [6, 9]. When $\Delta=\Delta_{F}$ and $G=P G L(3, F)$, the $\pi_{z}$ are irreducible and pairwise inequivalent. If $\Delta=\Delta_{\mathscr{T}}$, and if $\Gamma_{\mathscr{T}}$ embeds as a lattice in $P G L(3, F)$, the representations $\pi_{z}$ are all irreducible and pairwise inequivalent by a recent result of Cowling and Steger [4]. In Section 6, we show that the weak closure of $\mathscr{A}$ in the von Neumann algebra $\mathscr{L}$ induced by the left regular representation on $\ell^{2}\left(\Gamma_{\mathscr{T}}\right)$ is maximal abelian in $\mathscr{L}$. This implies that almost every $\pi_{z}$ is irreducible, even when $\Gamma_{\mathscr{T}}$ cannot be embedded as a lattice in $P G L(3, F)$ (see [9]).

We would like to thank Tim Steger for suggesting the problem solved in Section 6, and also both Michael Cowling and him for some useful comments. When this paper was in its final stages of preparation, we were informed that Anna Maria Mantero and Anna Zappa had independently obtained some of our results.

\section{The algebra $\mathscr{A}$. Biradial functions}

We continue using the notation of Section 1 . We start by considering $v \in$ $S_{j, k}(u)$ and $w \in S_{1,0}(v)$ (respectively $w \in S_{0,1}(v)$ ), and giving the possible $(m, n)$ such that $w \in S_{m, n}(u)$.

LEMMA 2.1. Let $v \in S_{j, k}(u)$ and $w \in S_{1,0}(v)$ (respectively, $w \in S_{0,1}(v)$ ). Then

(a) If $j, k \geq 1$, then

$$
w \in\left\{\begin{array} { l } 
{ S _ { j + 1 , k } ( u ) \text { for } q ^ { 2 } w ' s } \\
{ S _ { j - 1 , k + 1 } ( u ) \text { for } q \text { w's } } \\
{ S _ { j , k - 1 } ( u ) \text { for } 1 w }
\end{array} \quad \text { (respectively) } w \in \left\{\begin{array}{l}
S_{j, k+1}(u) \text { for } q^{2} w^{\prime} s \\
S_{j+1, k-1}(u) \text { for } q w^{\prime} s \\
S_{j-1, k}(u) \text { for } 1 w .
\end{array}\right.\right.
$$

(b) If $j=0$ and $k \geq 1$, then

$$
w \in\left\{\begin{array} { l } 
{ S _ { 1 , k } ( u ) \text { for } q ^ { 2 } + q w ^ { \prime } s } \\
{ S _ { 0 , k - 1 } ( u ) \text { for } 1 w }
\end{array} \quad \text { (respectively) } w \in \left\{\begin{array}{l}
S_{0, k+1}(u) \text { for } q^{2} w^{\prime} s \\
S_{1, k-1}(u) \text { for } q+1 w^{\prime} s
\end{array}\right.\right.
$$


(c) If $j \geq 1$ and $k=0$, then

$w \in\left\{\begin{array}{l}S_{j+1,0}(u) \text { for } q^{2} w^{\prime} s \\ S_{j-1,1}(u) \text { for } q+1 w^{\prime} s\end{array} \quad(\right.$ respectively $) w \in\left\{\begin{array}{l}S_{j, 1}(u) \text { for } q^{2}+q w^{\prime} s \\ S_{j-1,0}(u) \text { for } 1 w .\end{array}\right.$

PROOF. Consider Figure 1, with the $(m, n)$ there replaced by $(j, k)$, and let $x_{0}=v^{\prime}, \ldots, x_{k}=v$ be the vertices of the segment $v^{\prime} v$, and let $y_{0}=v^{\prime \prime}, \ldots, y_{j}=$ $v$ be the vertices of the segment $v^{\prime \prime} v$.

Suppose first that $j, k \geq 1$. Let $v$ have type $\tau(v)=0$, say. There are $q+1$ chambers containing the edge $\left\{y_{j-1}, v\right\}$, and one of these is $\left\{y_{j-1}, v, x_{k-1}\right\}$. Note that $\tau\left(y_{j-1}\right)=2$ and that $\tau\left(x_{k-1}\right)=1$. Clearly $x_{k-1} \in S_{j, k-1}(u)$ and $x_{k-1} \in S_{1,0}(v)$. For each of the remaining $q$ chambers containing $\left\{y_{j-1}, v\right\}$, its type 1 vertex $w$ is in $S_{j-1, k+1}(u)$. For the convex hull of $u$ and $w$ consists of that of $u$ and $y_{j-1}$ together with $2(j-1)$ new chambers, two containing $y_{i}$ for each $i=1, \ldots, j-1$ (see Figure 2(a)). For working down from $y_{j-1}$, let $x$ be the unique type 0 neighbour of $y_{j-1}$ which is a neighbour of both $w$ and $y_{j-1}$.

The two new chambers containing $y_{j-1}$ are $\left\{y_{j-1}, w, x\right\}$ and $\left\{y_{j-1}, y_{j-2}, x\right\}$. The other new chambers are constructed in a similar way. Now let $\left\{v, x_{k-1}, w^{\prime}\right\}$ be a chamber, other than $\left\{v, x_{k-1}, y_{j-1}\right\}$, containing $\left\{v, x_{k-1}\right\}$. Note that $\tau\left(w^{\prime}\right)=$ 2. For each of the $q$ chambers other than $\left\{v, w^{\prime}, x_{k-1}\right\}$ containing $\left\{v, w^{\prime}\right\}$, its type 1 vertex $w$ is in $S_{1,0}(v)$ and in $S_{j+1, k}(u)$ for similar reasons to those above (see Figure 2(b)). Also, for each such $w$, the vertex $w^{\prime}$ is determined, being the unique type 2 vertex which is a neighbour of $v$ and of both $w$ and $x_{k-1}$. So there are $q^{2}$ such $w$ 's. This proves the first part of (a).

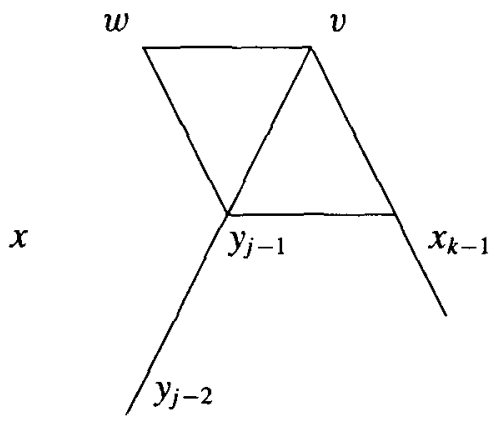

(a)

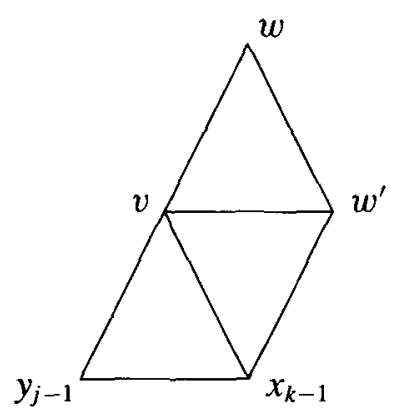

(b)

FIGURE 2 


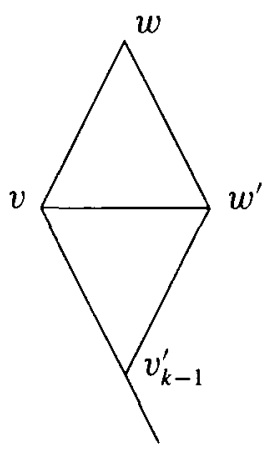

(a)

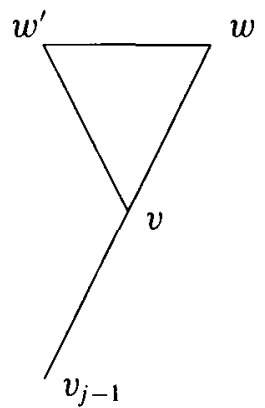

(b)

FIGURE 3

When $j=0$ and $k \geq 1$, the picture is a little different. Assuming once again that $v$ have type $\tau(v)=0$, say, the vertex $v_{k-1}^{\prime}$ is in $S_{1,0}(v)$ and $S_{0, k-1}(u)$. For each of the $q+1$ vertices $w^{\prime}$ lying on a chamber containing $\left\{v, v_{k-1}^{\prime}\right\}$, there are $q$ vertices $w$ lying on a chamber other than $\left\{v, w^{\prime}, v_{k-1}^{\prime}\right\}$ containing the edge $\left\{v, w^{\prime}\right\}$ (see Figure 3(a)). Each such $w$ is in $S_{1, k}(u)$ and $S_{1,0}(v)$, and there are $q(q+1)$ of them. This proves the first part of (b).

When $j \geq 1$ and $k=0$, each of the $q+1$ type 1 vertices $w$ lying in a chamber containing $\left\{v_{j-1}, v\right\}$ is in $S_{1,0}(v)$ and $S_{j-1,1}(u)$. For each of the $q^{2}$ remaining type 1 neighbours of $v$, pick any type 2 vertex $w^{\prime}$ lying on a chamber containing $\{v, w\}$. Then $w \neq v_{j-1}$, and by similar reasoning to that above, we see that $w^{\prime} \in S_{j, 1}(u)$ and that $w \in S_{j+1,0}$ (see Figure 3(b)). This proves the first part of (c).

The second parts of (a), (b) and (c) may be proved in the same way.

COROLlaRY 2.2. The cardinalities $N_{m, n}=\left|S_{m, n}(u)\right|$ do not depend on $u$, satisfy $N_{m, n}=N_{n, m}$, and are as follows:

$$
\begin{aligned}
& N_{0,0}=1, \\
& N_{m, 0}=N_{0, m}=\left(q^{2}+q+1\right) q^{2(m-1)} \quad \text { if } m \geq 1, \\
& N_{m, n}=\left(q^{2}+q+1\right)\left(q^{2}+q\right) q^{2(m+n-2)} \quad \text { if } m, n \geq 1 .
\end{aligned}
$$

PROOF. We show that

(i) $\left|S_{m+1, n}(u)\right|=q^{2}\left|S_{m, n}(u)\right|$ if $m \geq 1$ and $n \geq 0$, 
(ii) $\left|S_{m, n+1}(u)\right|=q^{2}\left|S_{m, n}(u)\right|$ if $m \geq 0$ and $n \geq 1$,

(iii) $\left|S_{1, n}(u)\right|=\left(q^{2}+q\right)\left|S_{0, n}(u)\right|$ if $n \geq 1$, and

(iv) $\left|S_{m, 1}(u)\right|=\left(q^{2}+q\right)\left|S_{m, 0}(u)\right|$ if $m \geq 1$.

Then the result follows from $\left|S_{1,0}(u)\right|=\left|S_{0,1}(u)\right|=q^{2}+q+1$ and induction.

To see (i), if $v \in S_{m+1, n}(u)$, let $f(v)$ be the unique $w \in S_{m, n}(u)$ such that $w \in S_{0,1}(v)$. If $w \in S_{m, n}(u)$, where $m \geq 1$, then $v \in S_{1,0}(w)$, that is, $w \in S_{0,1}(v)$, for $q^{2} v$ 's in $S_{m+1, n}(u)$. Thus $v \mapsto f(v)$ is a $q^{2}$-to-1 map, and (i) is proved. The other formulas are proved in a similar way.

Let $A^{+}\left(=A_{1,0}\right), A^{-}\left(=A_{0,1}\right)$ and $A_{m, n}$ be the averaging operators defined in Section 1.

PROPOSITION 2.3. The linear span $\mathscr{A}$ of the operators $A_{m, n}$ is a commutative algebra with identity $I=A_{0,0}$, and is generated by $A^{+}$and $A^{-}$. Moreover, the following formulas hold:

$$
\begin{aligned}
& A_{0,0} A^{+}=A_{1,0} \\
& A_{0, n} A^{+}=\frac{\left(q^{2}+q\right) A_{1, n}+A_{0, n-1}}{q^{2}+q+1} \quad \text { if } n \geq 1 \\
& A_{m, 0} A^{+}=\frac{q^{2} A_{m+1,0}+(q+1) A_{m-1,1}}{q^{2}+q+1} \quad \text { if } m \geq 1 \\
& A_{m, n} A^{+}=\frac{q^{2} A_{m+1, n}+q A_{m-1, n+1}+A_{m, n-1}}{q^{2}+q+1} \quad \text { if } m, n \geq 1
\end{aligned}
$$

$A_{0,0} A^{-}=A_{0,1}$

b $\left.^{\prime}\right) \quad A_{m, 0} A^{-}=\frac{\left(q^{2}+q\right) A_{m, 1}+A_{m-1,0}}{q^{2}+q+1} \quad$ if $m \geq 1$

$A_{0, n} A^{-}=\frac{q^{2} A_{0, n+1}+(q+1) A_{1, n-1}}{q^{2}+q+1} \quad$ if $n \geq 1$

(d') $\quad A_{m, n} A^{-}=\frac{q^{2} A_{m, n+1}+q A_{m+1, n-1}+A_{m-1, n}}{q^{2}+q+1} \quad$ if $m, n \geq 1$

PROOF. For a function $f$ on $\mathscr{V}$,

$$
\left(A_{m, n} A^{+} f\right)(u)=\frac{1}{\left(q^{2}+q+1\right) N_{m, n}} \sum_{w \in S_{m, n}(u)} \sum_{v \in S_{1,0}(w)} f(v)
$$




$$
=\frac{1}{\left(q^{2}+q+1\right) N_{m, n}} \sum_{v \in \mathscr{V}} N_{v} f(v)
$$

where

$$
N_{v}=\sharp\left\{w \in S_{m, n}(u): v \in S_{1,0}(w)\right\}=\sharp\left\{w \in S_{m, n}(u): w \in S_{0,1}(v)\right\} .
$$

These numbers are given in Lemma 2.1. Thus if $v \in S_{j, k}(u)$, then $N_{v}>0$ only for $(m, n)=(j, k+1),(j+1, k-1)$ or $(j-1, k)$, that is, $(j, k)=(m, n-1)$, $(m-1, n+1)$ or $(m+1, n)$, where the numbers in each pair must be nonnegative, of course. If $m, n \geq 2$, then each possible $(j, k)$ satisfies $j, k \geq 1$, and $N_{v}=q^{2}$, $q$ and 1 , respectively. Thus

$$
\begin{aligned}
\sum_{v \in \mathscr{V}} N_{v} f(v) & =q^{2} \sum_{v \in S_{m, n-1}(u)} f(v)+q \sum_{v \in S_{m-1, n+1}(u)} f(v)+\sum_{v \in S_{m+1, n}(u)} f(v) \\
& =N_{m, n}\left(A_{m, n-1} f(u)+q A_{m-1, n+1} f(u)+q^{2} A_{m+1, n} f(u)\right) .
\end{aligned}
$$

This proves (d) when $m, n \geq 2$. If $m \geq 2$ and $n=1$, then $k=0$ for the pair $(j, k)=(m, n-1)$, and so $N_{v}=q^{2}+q$ if $v \in S_{m, 0}$. Thus

$$
\sum_{v \in S_{m, 0}(u)} N_{v} f(v)=\left(q^{2}+q\right) N_{m, 0} A_{m, 0} f(u)=N_{m, 1}\left(A_{m, 0} f\right)(u) .
$$

This yields (d) when $m \geq 2$ and $n=1$. The other cases of (d), and the other formulas, are proved in the same way.

Now $A^{+}$and $A^{-}$commute, by special cases of (b) and ( $\left.b^{\prime}\right)$. By a simple induction on $m+n$, we see that each $A_{m, n}$ is a polynomial in $A^{+}$and $A^{-}$. Similarly, by induction on $k$, any product $A^{\epsilon_{1}} \cdots A^{\epsilon_{k}}$, where each $\epsilon_{j}$ is + or is a linear combination of the $A_{m, n}$. This proves the first statement in the Proposition.

Biradial functions Let $(f, g)=\sum_{v \in \mathcal{V}} f(v) g(v)$ whenever the sum on the right converges absolutely. If $A \in \mathscr{A}$, let $A^{*}$ be the adjoint of $A$ with respect to this bilinear form: $(A f, g)=\left(f, A^{*} g\right)$. Since $A_{m, n}^{*}=A_{n, m}$, we see that $A^{*} \in \mathscr{A}$ whenever $A \in \mathscr{A}$.

Let $o$ be a fixed vertex of $\Delta$. Let $\chi_{m, n}$ denote the characteristic function of $S_{m, n}(o)$. Using the fact that $v \in S_{m, n}(u)$ if and only if $u \in S_{n, m}(v)$ (and $\left.N_{m, n}=N_{n, m}\right)$, we see that

$$
A_{m, n} \delta_{o}=\frac{\chi_{n, m}}{N_{n, m}}
$$


We shall call functions which are constant on each set $S_{m, n}(o)(o$-)biradial. The finitely supported biradial functions have the $\chi_{m, n}, m, n \in \mathbb{N}$, as a basis. Thus $A \mapsto A^{*} \delta_{o}$ gives a vector space isomorphism of $\mathscr{A}$ onto this space of functions, mapping $A_{m, n}$ to $\chi_{m, n} / N_{m, n}$.

Notice that $A\left(B \delta_{o}\right)=(A B) \delta_{o}$ implies that each $A \in \mathscr{A}$ leaves invariant the space of biradial functions of finite support, and hence the space of all biradial functions. If $\varphi$ is the biradial function $\sum_{m, n} \alpha_{m, n} \chi_{m, n}$, we define $\varphi^{*}$ to be the biradial function $\sum_{m, n} \alpha_{n, m} \chi_{m, n}$. Note that for $A \in \mathscr{A}$, and biradial $\varphi$,

$$
A^{*} \varphi^{*}=(A \varphi)^{*} .
$$

This holds because $\mathscr{A}$ is abelian and, when $\varphi$ is the finitely supported biradial function corresponding to $B \in \mathscr{A}$ under the above isomorphism, $\varphi^{*}$ is that corresponding to $B^{*}$.

For any function $f$ on $\mathscr{V}$, let

$$
(\mathscr{E} f)(x)=\frac{1}{N_{m, n}} \sum_{y \in S_{m, n}(o)} f(y) \quad\left(\text { if } x \in S_{m, n}(o)\right) .
$$

Then $\mathscr{E} f$ is biradial, and $\mathscr{E}$ is a projection onto the space of biradial functions. Notice that $\mathscr{E}^{*}=\mathscr{E}$, so that if $g$ is biradial, then $(f, g)=(\mathscr{E} f, g)$, for any function $f$ of finite support. Also, $\mathscr{E}$ commutes with each $A \in \mathscr{A}$ : if $f$ and $g$ have finite support, then

$$
\begin{aligned}
(A \mathscr{E} f, g) & =(A \mathscr{E} f, \mathscr{E} g) \quad \text { as } A \mathscr{E} f \text { is biradial } \\
& =\left(\mathscr{E} f, A^{*} \mathscr{E} g\right) \\
& =\left(f, A^{*} \mathscr{E} g\right) \quad \text { as } A^{*} \mathscr{E} g \text { is biradial } \\
& =(A f, \mathscr{E} g) \\
& =(\mathscr{E} A f, g)
\end{aligned}
$$

When $\Delta=\Delta_{\mathscr{T}}$, we can take $o$ to be the identity element 1 of $\Gamma_{\mathscr{T}}$. If we let

$$
\mu^{+}=\frac{1}{q^{2}+q+1} \sum_{x \in P} \delta_{a_{x}} \quad \text { and } \quad \mu^{-}=\frac{1}{q^{2}+q+1} \sum_{x \in P} \delta_{a_{x}^{-1}}
$$

then $A^{+} f=f * \mu^{-}$and $A^{-} f=f * \mu^{+}$, and the biradial functions of finite support form a convolution algebra isomorphic to $\mathscr{A}$.

The next proposition gives another interpretation of $\mathscr{A}$ when $\Delta=\Delta_{F}$, for example. 
Let $G$ be a subgroup of $\operatorname{Aut}_{\mathrm{tr}}(\Delta)$ which is closed for the topology of pointwise convergence. The subgroup $K=\{g \in G: g o=o\}$ is compact and open for this topology. Notice that $K$ acts on each set $S_{m, n}(o)$. Taking a left Haar measure on $G$ normalized so that $K$ has measure 1 , the space $\mathscr{L}(G, K)$ of compactly supported bi- $K$-invariant functions on $G$ is a convolution algebra, and is spanned by the functions $\chi_{K g K}, g \in G$ (see, for example, [7, Proposition 1.1.1]).

PROPOSITION 2.4. Assume that $G$ acts transitively on $\mathscr{V}$, and that $K$ acts transitively on each set $S_{m, n}(o)$. Then $\mathscr{L}(G, K)$ is isomorphic to $\mathscr{A}$ under the map determined by $\chi_{K_{g} K} \mapsto N_{m, n} A_{m, n}$ (if go $\in S_{m, n}(o)$ ). Thus $(G, K)$ is a Gelfand pair.

PROOF. The hypotheses imply that if $g, g^{\prime} \in G, g o \in S_{m, n}(o)$ and $g^{\prime} o \in$ $S_{r, s}(o)$, then $K g K=K g^{\prime} K$ if and only if $(m, n)=(r, s)$. For each $j, k \in \mathbb{N}$, pick $g_{j, k} \in G$ such that $g_{j, k} o \in S_{j, k}(o)$. If $g o \in S_{m, n}(o)$ and $g^{\prime} o \in S_{r, s}(o)$, then

$$
\chi_{K g K} * \chi_{K g^{\prime} K}=\sum_{j, k} c_{j, k} \chi_{K g_{j, k} K}
$$

where

$$
\begin{aligned}
c_{j, k} & =\left(\chi_{K g K} * \chi_{K g^{\prime} K}\right)\left(g_{j, k}\right) \\
& =\int_{G} \chi_{K g K}\left(g_{j, k} h\right) \chi_{K g^{\prime} K}\left(h^{-1}\right) d h .
\end{aligned}
$$

Break this integral up into the sum of integrals over the distinct cosets $g_{v} K=$ $\{h \in G: h o=v\}, v \in \mathscr{V}$, where $g_{v} \in G$ satisfies $g_{v} o=v$. For each $v \in \mathscr{V}$, the integrand is constant on $g_{v} K$, being either always 1 there if $g_{j, k} g_{v} \in K g K$ and $g_{v}^{-1} \in K g^{\prime} K$, or always 0 there. Note that

$g_{v}^{-1} \in K g^{\prime} K \quad$ if and only if $\quad g_{v}^{-1} o \in S_{r, s}(o)$

$$
\text { if and only if } o \in S_{r, s}(v) \text { if and only if } v \in S_{s, r}(o)
$$

and similarly $g_{j, k} g_{v} \in K g K$ if and only if $v \in S_{m, n}\left(g_{j, k}^{-1} o\right)$. Thus $c_{j, k}=$ $\left|S_{m, n}\left(g_{j, k}^{-1} o\right) \cap S_{s, r}(o)\right|$. Notice that $g_{j, k}^{-1} o \in S_{k, j}(o)$. On the other hand, we can write

$$
\left(N_{m, n} A_{m, n}\right)\left(N_{r, s} A_{r, s}\right)=\sum_{\alpha, \beta} c_{\alpha, \beta}^{\prime}\left(N_{\alpha, \beta} A_{\alpha, \beta}\right)
$$

for suitable constants $c_{\alpha, \beta}^{\prime}$. If we apply both sides of this to $\delta_{o}$ and evaluate at any $u \in S_{k, j}(o)$, we immediately obtain $c_{j, k}^{\prime}=\left|S_{m, n}(u) \cap S_{s, r}(o)\right|=c_{j, k}$. This proves the result. 
EXAMPLE. In the notation of Section 1, if $\Delta=\Delta_{F}$, the last proposition applies if we take $G=\mathscr{G}$ and $K=\mathscr{K}$. If we let $G=S L(3, F)$ and $K=S L(3, \mathscr{O}), G$ does not act transitively on $\mathscr{V}$, and $\mathscr{L}(G, K)$ is isomorphic to the subalgebra $\mathscr{A}_{0}$ of $\mathscr{A}$ defined in the next result.

PROPOSITION 2.5. Let $\mathscr{A}_{0}$ be the subspace of $\mathscr{A}$ spanned by the $A_{m, n}$ for which $m-n \equiv 0(\bmod 3)$. Then $\mathscr{A}_{0}$ is a subalgebra of $\mathscr{A}$, and is generated by $A^{+} A^{-},\left(A^{+}\right)^{3}$ and $\left(A^{-}\right)^{3}$.

PROOF. Let $\omega=e^{2 \pi i / 3}$. It is immediate from the formulas in Proposition 2.3 that there is an algebra isomorphism $\Phi$ of $\mathscr{A}$ such that $\Phi\left(A_{m, n}\right)=\omega^{m-n} A_{m, n}$. Clearly $\mathscr{A}_{0}=\{A \in \mathscr{A}: \Phi(A)=A\}$, and so is a subalgebra of $\mathscr{A}$. It is evident from this and the formulas in Proposition 2.3 that if $m-n \equiv 0(\bmod 3)$, then for some number $c=c_{m, n}$,

$$
\begin{aligned}
& A^{+} A^{-} A_{m, n}=c A_{m+1, n+1}+\text { terms in } A_{j, k}, \\
& \text { where } j+k<m+n+2 \text { and } j-k \equiv 0(\bmod 3) .
\end{aligned}
$$

Similar formulas hold for $\left(A^{+}\right)^{3} A_{m, n}$ and $\left(A^{-}\right)^{3} A_{m, n}$. A simple induction now shows that $\mathscr{A}_{0}$ is generated by $A^{+} A^{-},\left(A^{+}\right)^{3}$ and $\left(A^{-}\right)^{3}$.

\section{The multiplicative functionals on $\mathscr{A}$. Spherical functions.}

In this section, we show that for each $z, w \in \mathbb{C}$ there is a multiplicative functional $h=h_{z, w}$ on $\mathscr{A}$ such that $z=h\left(A^{+}\right)$and $w=h\left(A^{-}\right)$, which is clearly unique. We then explicitly calculate

$$
p_{m, n}(z, w)=h_{z, w}\left(A_{m, n}\right) .
$$

Once the existence of $h_{z, w}$ has been demonstrated, it is evident from the formulas in Proposition 2.3 that each $p_{m, n}(z, w)$ is a polynomial in $z$ and $w$.

It turns out that the multiplicative functionals may be indexed by the group $S=\left\{s=\left(s_{1}, s_{2}, s_{3}\right) \in \mathbb{C}^{3}: s_{1} s_{2} s_{3}=1\right\}$, the correspondence between $(z, w)$ and $s=\left(s_{1}, s_{2}, s_{3}\right)$ being given by

$$
z=\frac{q}{q^{2}+q+1}\left(s_{1}+s_{2}+s_{3}\right) \quad \text { and } \quad w=\frac{q}{q^{2}+q+1}\left(\frac{1}{s_{1}}+\frac{1}{s_{2}}+\frac{1}{s_{3}}\right)
$$


and we sometimes write $h_{s}$ in place of $h_{z, w}$, when $s=\left(s_{1}, s_{2}, s_{3}\right)$ is related in this way to $(z, w)$. Notice that if $s=\left(s_{1}, s_{2}, s_{3}\right) \in S$ and $s^{\prime}=\left(s_{1}^{\prime}, s_{2}^{\prime}, s_{3}^{\prime}\right) \in S$, then $h_{s^{\prime}}=h_{s}$ if and only if $\left(s_{1}^{\prime}, s_{2}^{\prime}, s_{3}^{\prime}\right)=\left(s_{\sigma 1}, s_{\sigma 2}, s_{\sigma 3}\right)$ for some permutation $\sigma$ of $\{1,2,3\}$.

Let $z, w \in \mathbb{C}$. We wish to solve the equations

$$
\begin{aligned}
p_{0,0} & =1, \quad p_{1,0}=z, \quad p_{0,1}=w \\
z p_{m, n} & =\frac{q^{2} p_{m+1, n}+q p_{m-1, n+1}+p_{m, n-1}}{q^{2}+q+1} \quad(m, n \geq 1) \\
w p_{m, n} & =\frac{q^{2} p_{m, n+1}+q p_{m+1, n-1}+p_{m-1, n}}{q^{2}+q+1} \quad(m, n \geq 1) \\
z p_{m, 0} & =\frac{q^{2} p_{m+1,0}+(q+1) p_{m-1,1}}{q^{2}+q+1} \quad(m \geq 1) \\
w p_{m, 0} & =\frac{\left(q^{2}+q\right) p_{m, 1}+p_{m-1,0}}{q^{2}+q+1} \quad(m \geq 1) \\
z p_{0, n} & =\frac{\left(q^{2}+q\right) p_{1, n}+p_{0, n-1}}{q^{2}+q+1} \quad(n \geq 1) \\
w p_{0, n} & =\frac{q^{2} p_{0, n+1}+(q+1) p_{1, n-1}}{q^{2}+q+1} \quad(n \geq 1)
\end{aligned}
$$

Writing $z^{\prime}=\left(q^{2}+q+1\right) z / q, w^{\prime}=\left(q^{2}+q+1\right) w / q$ and $p_{m, n}^{\prime}=q^{m+n} p_{m, n}$, these equations become

$$
\begin{aligned}
p_{0,0}^{\prime} & =1, \quad p_{1,0}^{\prime}=\frac{q^{2} z^{\prime}}{\left(q^{2}+q+1\right)}, \quad p_{0,1}^{\prime}=\frac{q^{2} w^{\prime}}{\left(q^{2}+q+1\right)} \\
z^{\prime} p_{m, n}^{\prime} & =p_{m+1, n}^{\prime}+p_{m-1, n+1}^{\prime}+p_{m, n-1}^{\prime} \quad(m, n \geq 1) \\
w^{\prime} p_{m, n}^{\prime} & =p_{m, n+1}^{\prime}+p_{m+1, n-1}^{\prime}+p_{m-1, n}^{\prime} \quad(m, n \geq 1) \\
z^{\prime} p_{m, 0}^{\prime} & =p_{m+1,0}^{\prime}+\frac{q+1}{q} p_{m-1,1}^{\prime} \quad(m \geq 1) \\
w^{\prime} p_{m, 0}^{\prime} & =\frac{q+1}{q} p_{m, 1}^{\prime}+p_{m-1,0}^{\prime} \quad(m \geq 1) \\
z^{\prime} p_{0, n}^{\prime} & =\frac{q+1}{q} p_{1, n}^{\prime}+p_{0, n-1}^{\prime} \quad(n \geq 1) \\
w^{\prime} p_{0, n}^{\prime} & =p_{0, n+1}^{\prime}+\frac{q+1}{q} p_{1, n-1}^{\prime} \quad(n \geq 1)
\end{aligned}
$$


Using $3.4^{\prime}$ and $3.5^{\prime}$, we see that $p_{m, 0}^{\prime}$ satisfies the relation

$$
p_{m+2,0}^{\prime}-z^{\prime} p_{m+1,0}^{\prime}+w^{\prime} p_{m, 0}^{\prime}-p_{m-1,0}^{\prime}=0 \quad(m \geq 1) .
$$

Consider the cubic equation

$$
X^{3}-z^{\prime} X^{2}+w^{\prime} X-1=0 .
$$

Assume that the roots of (3.9) are distinct, and denote them by $s_{i}, i=1,2,3$. Then the solution of (3.8) is

$$
p_{m, 0}^{\prime}=A_{1,0} s_{1}^{m}+A_{2,0} s_{2}^{m}+A_{3,0} s_{3}^{m} \quad(m \geq 0)
$$

where $A_{i, 0}, i=1,2,3$, are the unique numbers satisfying (3.10) for $m=0,1,2$. Solving, one finds that

$$
A_{i, 0}=\frac{\left(s_{i} q-s_{i^{\prime}}\right)\left(s_{i} q-s_{i^{\prime \prime}}\right)}{\left(s_{i}-s_{i^{\prime}}\right)\left(s_{i}-s_{i^{\prime \prime}}\right)\left(q^{2}+q+1\right)} \quad(i=1,2,3)
$$

where $i^{\prime}, i^{\prime \prime}$ denote the numbers in $\{1,2,3\}$ other than $i$.

We see from $3.4^{\prime}$ that

$$
p_{m, 1}^{\prime}=A_{1,1} s_{1}^{m}+A_{2,1} s_{2}^{m}+A_{3,1} s_{3}^{m} \quad(m \geq 0)
$$

where

$$
A_{i, 1}=\frac{q}{q+1} s_{i}\left(z^{\prime}-s_{i}\right) A_{i, 0}=\frac{q}{q+1}\left(\frac{1}{s_{i^{\prime}}}+\frac{1}{s_{i^{\prime \prime}}}\right) A_{i, 0}
$$

(with the same meaning for $i^{\prime}$ and $i^{\prime \prime}$ ). Using 3.2', with $m+1$ in place of $m$, we see that

$$
p_{m, n}^{\prime}=A_{1, n} s_{1}^{m}+A_{2, n} s_{2}^{m}+A_{3, n} s_{3}^{m} \quad(m \geq 0)
$$

where

$$
A_{i, n+1}=s_{i}\left(z^{\prime}-s_{i}\right) A_{i, n}-s_{i} A_{i, n-1} \quad(n \geq 1) .
$$

Now the roots of the quadratic equation

$$
X^{2}-s_{i}\left(z^{\prime}-s_{i}\right) X+s_{i}=0
$$

are $X=1 / s_{i^{\prime}}$ and $X=1 / s_{i^{\prime \prime}}$. Thus

$$
A_{i, n}=\frac{C_{i, i^{\prime}}}{s_{i^{\prime}}^{n}}+\frac{C_{i, i^{\prime \prime}}}{s_{i^{\prime \prime}}^{n}} \quad(n \geq 0)
$$


where $C_{i, i^{\prime}}$ and $C_{i, i^{\prime \prime}}$ are the unique numbers such that (3.14) holds for $n=0,1$. It follows that

$$
p_{m, n}^{\prime}=\sum_{i, j: i \neq j} C_{i, j} \frac{s_{i}^{m}}{s_{j}^{n}}
$$

When we solve (3.14) for $n=0,1$, we find that

$$
C_{i, j}=\frac{q^{3}}{(q+1)\left(q^{2}+q+1\right)} c\left(s_{\sigma 1}, s_{\sigma 2}, s_{\sigma 3}\right)
$$

for a permutation $\sigma=\sigma_{i, j}$ of $\{1,2,3\}$, where

$$
c\left(s_{1}, s_{2}, s_{3}\right)=\prod_{i<j} \frac{s_{i}-\frac{1}{q} s_{j}}{s_{i}-s_{j}}=\left(\frac{s_{1}-\frac{1}{q} s_{2}}{s_{1}-s_{2}}\right)\left(\frac{s_{1}-\frac{1}{q} s_{3}}{s_{1}-s_{3}}\right)\left(\frac{s_{2}-\frac{1}{q} s_{3}}{s_{2}-s_{3}}\right) .
$$

Indeed, $\sigma_{1,2}=(2,3), \sigma_{1,3}=i d, \sigma_{2,1}=(1,2,3), \sigma_{2,3}=(1,2), \sigma_{3,1}=(1,3)$ and $\sigma_{3,2}=(1,3,2)$. Moreover, if we take integers $n_{1}, n_{2}$ and $n_{3}$ so that $m=n_{1}-n_{2}$ and $n=n_{2}-n_{3}$, we find that $s_{i}^{m} / s_{j}^{n}=s_{\sigma 1}^{n_{1}} s_{\sigma 2}^{n_{2}} s_{\sigma 3}^{n_{3}}$ for $\sigma=\sigma_{i, j}$. Notice that $n_{1} \geq n_{2} \geq n_{3}$, and that $s_{\sigma 1}^{n_{1}} s_{\sigma 2}^{n_{2}} s_{\sigma 3}^{n_{3}}$ is unchanged if we replace $\left(n_{1}, n_{2}, n_{3}\right)$ by $\left(n_{1}+k, n_{2}+k, n_{3}+k\right)$ for any integer $k$ (because $\left.s_{1} s_{2} s_{3}=1\right)$.

If, conversely, the $p_{m, n}^{\prime}$ are given by (3.15) for these $C_{i, j}$, then it is routine to check that they satisfy conditions $3.1^{\prime}-3.7^{\prime}$ above.

We have therefore proved the following result:

PROPOSITION 3.1. Suppose that

$$
z=\frac{q}{q^{2}+q+1}\left(s_{1}+s_{2}+s_{3}\right) \text { and } w=\frac{q}{q^{2}+q+1}\left(\frac{1}{s_{1}}+\frac{1}{s_{2}}+\frac{1}{s_{3}}\right)
$$

where $s_{1} s_{2} s_{3}=1$. Suppose that $s_{1}, s_{2}$ and $s_{3}$ are distinct. Then, writing $S_{3}$ for the group of permutations of $\{1,2,3\}$,

$$
p_{m, n}(z, w)=\frac{q^{3}}{(q+1)\left(q^{2}+q+1\right)} \frac{1}{q^{m+n}} \sum_{\sigma \in S_{3}} s_{\sigma 1}^{n_{1}} s_{\sigma 2}^{n_{2}} s_{\sigma 3}^{n_{3}} c\left(s_{\sigma 1}, s_{\sigma 2}, s_{\sigma 3}\right)
$$

for any integers $n_{1}, n_{2}, n_{3}$ such that $m=n_{1}-n_{2}$ and $n=n_{2}-n_{3}$.

Remarks. Let $\mathscr{A}_{0}$ be the subalgebra of $\mathscr{A}$ defined in Proposition 2.5. Consider the restriction $h_{1 \infty_{0}}$ of $h=h_{z, w}$ to $\mathscr{A}_{0}$. If $h^{\prime}=h_{z^{\prime}, w^{\prime}}$, then $h_{1 \otimes_{0}}^{\prime}=h_{\mid \mathscr{A}_{0}}$ if and only if $\left(z^{\prime}, w^{\prime}\right)=(z, w),\left(\omega z, \omega^{-1} w\right)$ or $\left(\omega^{-1} z, \omega w\right)$, where $\omega=e^{2 \pi i / 3}$. Indeed, if $h_{\mid \otimes_{0}}^{\prime}=h_{1 \infty_{0}}$, then $\left(z^{\prime}\right)^{3}=h^{\prime}\left(\left(A^{+}\right)^{3}\right)=h\left(\left(A^{+}\right)^{3}\right)=z^{3},\left(w^{\prime}\right)^{3}=$ 
$h^{\prime}\left(\left(A^{-}\right)^{3}\right)=h\left(\left(A^{-}\right)^{3}\right)=w^{3}$ and $z^{\prime} w^{\prime}=h^{\prime}\left(A^{+} A^{-}\right)=h\left(A^{+} A^{-}\right)=z w$, so that $\left(z^{\prime}, w^{\prime}\right)=(z, w),\left(\omega z, \omega^{-1} w\right)$ or $\left(\omega^{-1} z, \omega w\right)$. The reverse implication follows from, for example, the identity $p_{m, n}\left(\omega z, \omega^{-1} w\right)=\omega^{m-n} p_{m, n}(z, w)$, which is easily proved by induction.

Each multiplicative functional $h_{0}$ on $\mathscr{A}_{0}$ is the restriction of a multiplicative functional $h$ on $\mathscr{A}$. For we can let $z$ be a cube root of $h_{0}\left(\left(A^{+}\right)^{3}\right)$ and $w$ a cube root of $h_{0}\left(\left(A^{-}\right)^{3}\right)$ so that $z w=h_{0}\left(A^{+} A^{-}\right)$. Then $h=h_{z, w}$ agrees with $h_{0}$ on $\left(A^{+}\right)^{3},\left(A^{-}\right)^{3}$ and $A^{+} A^{-}$, and hence on $\mathscr{A}_{0}$.

Formula (3.17) coincides with the formula in [7, p. 52] except that there $n_{1}+n_{2}+n_{3}=0$ and $s_{1}, s_{2}$ and $s_{3}$ do not satisfy $s_{1} s_{2} s_{3}=1$, and are determined only up to a common factor. Note that natural numbers $m$ and $n$ may be expressed $m=n_{1}-n_{2}$ and $n=n_{2}-n_{3}$ where $n_{1}+n_{2}+n_{3}=0$ if and only if $m-n \equiv 0$ (mod 3). Thus the formula in [7, p. 52] agrees with our formula for $h_{1 \infty_{0}}$.

The singular cases In the 'singular cases', that is, when the numbers $s_{j}$ are not distinct, we can solve the recurrence relations $\left(3.1^{\prime}-3.7^{\prime}\right)$ by appropriate modifications of the methods used above. However, it is quicker to appeal to the fact that $p_{m, n}$ must be a polynomial in the $s_{j}$ 's and their inverses, and obtain the desired formula by taking limits of the 'nonsingular formula'.

PROPOSITION 3.2. Suppose that $s_{2}=s_{3} \neq s_{1}$. Then for each $m, n \geq 0, p_{m, n}$ is given by

$$
\begin{aligned}
& \begin{aligned}
p_{m, n}(z, w)=\frac{1}{(q+1)\left(q^{2}+q+1\right)} \frac{1}{q^{m+n}} & \\
\times\left(\left(C_{1,2}+C_{1,2}^{\prime} n\right) s_{1}^{m} / s_{2}^{n}+\right. & \left(C_{2,1}+C_{2,1}^{\prime} m\right) s_{2}^{m} / s_{1}^{n} \\
& \left.+\left(C_{2,2}+C_{2,2}^{\prime}(m+n)\right) s_{2}^{m-n}\right)
\end{aligned} \\
& \begin{aligned}
(3.18) \quad
\end{aligned}
\end{aligned}
$$

where

$$
\begin{array}{ll}
C_{1,2}=\frac{(q+1)\left(s_{1} q-s_{2}\right)^{2}}{\left(s_{1}-s_{2}\right)^{2}} & C_{1,2}^{\prime}=\frac{(q-1)\left(s_{1} q-s_{2}\right)^{2}}{\left(s_{1}-s_{2}\right)^{2}} \\
C_{2,1}=\frac{(q+1)\left(s_{2} q-s_{1}\right)^{2}}{\left(s_{2}-s_{1}\right)^{2}} & C_{2,1}^{\prime}=\frac{(q-1)\left(s_{2} q-s_{1}\right)^{2}}{\left(s_{2}-s_{1}\right)^{2}} \\
C_{2,2}=\frac{q(q+1)\left(s_{1}^{2}+s_{2}^{2}\right)-2\left(q^{3}+1\right) s_{1} s_{2}}{\left(s_{1}-s_{2}\right)^{2}} & C_{2,2}^{\prime}=\frac{-(q-1)\left(s_{1} q-s_{2}\right)\left(s_{2} q-s_{1}\right)}{\left(s_{2}-s_{1}\right)^{2}}
\end{array}
$$

ProOF. Consider formula (3.17). Combining the terms corresponding to $\sigma=i d$ and $\sigma=(23)$, letting $s_{3} \rightarrow s_{2}$, and using $\lim _{y \rightarrow x}\left(x^{k} y^{l}-x^{l} y^{k}\right) /(x-y)=$ 
$(k-l) x^{k+l-1}$, we obtain the terms in $s_{1}^{m} / s_{2}^{n}$ in (3.18). Similarly, combining the terms corresponding to $\sigma=\left(\begin{array}{ll}1 & 3\end{array}\right)$ and $\sigma=\left(\begin{array}{ll}1 & 2\end{array} 3\right)$, we obtain the terms in $s_{2}^{m} / s_{1}^{n}$ in (3.18). The terms in $s_{2}^{m-n}$ are obtained in the same way by combining the remaining two terms in (3.17), though this case is a little more complicated.

PROPOSITION 3.3. If $(z, w)$ and $\left(s_{1}, s_{2}, s_{3}\right)$ are as in $(3.16)$, with $s_{1}=s_{2}=s_{3}$, then

$$
\begin{aligned}
p_{m, n}(z, w)= & \frac{s_{1}^{m-n}}{2(q+1)\left(q^{2}+q+1\right)} \frac{1}{q^{m+n}} \\
& \quad \times\left\{(q-1)^{3} m n(m+n)+(q-1)^{2}(q+1)\left(m^{2}+4 m n+n^{2}\right)\right. \\
& \left.\quad+3(q-1)(q+1)^{2}(m+n)+2(q+1)\left(q^{2}+q+1\right)\right\} \\
= & \frac{s_{1}^{m-n} Q(m, n)}{q^{m+n}}, \quad \text { say. }
\end{aligned}
$$

PROOF. This can most easily be obtained from the formula in Proposition 3.2, letting $s_{2} \rightarrow s_{1}$ and applying l'Hôpital's rule.

Spherical functions The multiplicative functionals on $\mathscr{A}$ can be expressed in terms of (zonal) spherical functions in a well known way. Fix a vertex $o \in \mathscr{V}$. A function $\varphi$ on $\mathscr{V}$ is called spherical if

(i) $\varphi$ is biradial with respect to $o$;

(ii) $\varphi(o)=1$;

(iii) For each $A \in \mathscr{A}$ there is a number $c_{A}$ such that $A \varphi=c_{A} \varphi$.

PROPOSITION 3.4. Let $\varphi$ be a biradial function on $\mathscr{V}$. Then $\varphi$ is spherical if and only if

$$
h(A)=\left(A^{*} \delta_{o}, \varphi\right)=(A \varphi)(o)
$$

defines a multiplicative functional on $\mathscr{A}$. Moreover, each multiplicative functional on $\mathscr{A}$ arises in this way.

ProOF. Let $\varphi$ be spherical, and define $h: \mathscr{A} \rightarrow \mathbb{C}$ by (3.19). Then

$$
c_{A}=(A \varphi)(o)=h(A) .
$$

Also, if $A, B \in \mathscr{A}$, then

$$
h(A B)=(A B \varphi)(o)=\left(A c_{B} \varphi\right)(o)=c_{B} h(A)=h(A) h(B) .
$$


If now $\varphi$ is biradial and if (3.19) defines a multiplicative functional on $\mathscr{A}$, then for $A \in \mathscr{A}$ and $x \in S_{m, n}(o)$ we have

$$
\begin{aligned}
(A \varphi)(x) & =\left(\delta_{x}, A \varphi\right)=\left(\mathscr{E} \delta_{x}, A \varphi\right) \\
& =\left(\frac{\chi_{m, n}}{N_{m, n}}, A \varphi\right) \\
& =\left(A_{n, m} \delta_{o}, A \varphi\right)=\left(\delta_{o}, A_{m, n} A \varphi\right) \\
& =\left(\delta_{o}, A_{m, n} \varphi\right)\left(\delta_{o}, A \varphi\right)=\left(\mathscr{E} \delta_{x}, \varphi\right)\left(\delta_{o}, A \varphi\right) \\
& =c_{A} \varphi(x) \quad \text { for } c_{A}=\left(\delta_{o}, A \varphi\right) .
\end{aligned}
$$

Also, $\varphi(o)=h(I)=1$, and so $\varphi$ is spherical.

Finally, if $h$ is any multiplicative functional on $\mathscr{A}$, if we set $\varphi(x)=h\left(A_{m, n}\right)$ for any $x \in S_{m, n}(o)$, a similar calculation shows that $\varphi$ is a spherical function satisfying (3.19).

The spherical function associated with $h_{z, w}=h_{s}$ is

$$
\varphi_{z, w}=\varphi_{s}=\sum_{m, n=0}^{\infty} p_{m, n}(z, w) \chi_{m, n} .
$$

We mention also that the spherical functions on $\mathscr{V}$ may be characterized by being the nonzero functions satisfying, for each $u \in \mathscr{V}$ and each $m, n \in \mathbb{N}$,

$$
\frac{1}{N_{m, n}} \sum_{w \in S_{m, n}(u)} \varphi(w)=\varphi(u) \varphi(v) \quad \text { if } v \in S_{m, n}(o) .
$$

If $G$ is a subgroup of $\operatorname{Aut}_{\text {tr }}(\Delta)$ which acts transitively on $\mathscr{V}$, then this condition is equivalent to

$$
\mathscr{E}\left(\lambda\left(g^{-1}\right) \varphi\right)=\varphi(g o) \varphi
$$

where $\lambda(g) f(v)=f\left(g^{-1} v\right)$.

As we have already mentioned when calculating $p_{m, n}$ in the singular cases, we can express $p_{m, n}$ as a polynomial in the $s_{j}$ 's and their inverses, and therefore (as $s_{1} s_{2} s_{3}=1$ ) as a polynomial in the $s_{j}$ 's alone. In fact, we can do this so that all the coefficients are positive (compare [7, $\left.\left(3.3 .8^{\prime}\right)\right]$ ):

PROPOSITION 3.5. Let $x \in S_{m, n}$. Then we can write

$$
\varphi_{s}(x)=\sum_{\mathbf{k}} c_{m, n ; \mathbf{k}} s_{1}^{k_{1}} s_{2}^{k_{2}} s_{3}^{k_{3}} \quad\left(\left(s_{1}, s_{2}, s_{3}\right) \in S\right)
$$


where the sum is over a finite set of triples $\boldsymbol{k}=\left(k_{1}, k_{2}, k_{3}\right)$ of positive integers, and where the coefficients $c_{m, n ; \mathbf{k}}$ are nonnegative. Consequently,

$$
\left|\varphi_{s}(x)\right| \leq \varphi_{|s|}(x)
$$

where $|s|=\left(\left|s_{1}\right|,\left|s_{2}\right|,\left|s_{3}\right|\right)$.

PROOF. Consider the generating function

$$
F(x, y)=\sum_{m, n=0}^{\infty} q^{m+n} p_{m, n}(z, w) x^{m} y^{n} .
$$

Using Proposition 3.1, with $n_{1}=m+n, n_{2}=n$ and $n_{3}=0$, we see that $F(x, y)=\frac{q^{3}}{(q+1)\left(q^{2}+q+1\right)} \sum_{\sigma \in S_{3}} c\left(s_{\sigma 1}, s_{\sigma 2}, s_{\sigma 3}\right)\left(\frac{s_{\sigma 1} x}{1-s_{\sigma 1} x}\right)\left(\frac{s_{\sigma 1} s_{\sigma 2} y}{1-s_{\sigma 1} s_{\sigma 2} y}\right)$.

Now let $a_{i}=s_{i} x /\left(1-s_{i} x\right)(i=1,2,3)$,

$$
b_{1}=\frac{s_{2} s_{3} y}{1-s_{2} s_{3} y} \quad b_{2}=\frac{s_{1} s_{3} y}{1-s_{1} s_{3} y} \quad b_{3}=\frac{s_{1} s_{2} y}{1-s_{1} s_{2} y}
$$

and $r_{0}=(q+1)\left(q^{2}+q+1\right), r_{1}=q^{2}(q+1), r_{2}=(q-1) q(q+1)$, $r_{3}=(q-1) q(2 q+1) / 3, r_{4}=q^{3}, r_{5}=(q-1)^{2}(q+1), r_{6}=(q-1) q(4 q-$ $1) / 6, r_{7}=(q-1) q^{2}, r_{8}=2(q-1)^{2} q / 3$ and $r_{9}=(q-1)^{3} / 3$. Then the proposition is immediate from the fact that the following expression, divided by $(q+1)\left(q^{2}+q+1\right)$, equals $F(x, y)$ :

$$
\begin{aligned}
r_{0} & +r_{1} \sum_{i}\left(a_{i}+b_{i}\right)+r_{2} \sum_{i<j}\left(a_{i} a_{j}+b_{i} b_{j}\right) \\
& +r_{3} \sum_{i} a_{i} b_{i}+r_{4} \sum_{i \neq j} a_{i} b_{j}+r_{5}\left(a_{1} a_{2} a_{3}+b_{1} b_{2} b_{3}\right) \\
& +r_{6} \sum_{i<j}\left(a_{i} a_{j}\left(b_{i}+b_{j}\right)+\left(a_{i}+a_{j}\right) b_{i} b_{j}\right)+r_{7}\left(\sum_{i<j, k \neq i, j}\left(a_{i} a_{j} b_{k}+a_{k} b_{i} b_{j}\right)\right) \\
& +r_{8}\left(a_{1} a_{2} a_{3} \sum_{i} b_{i}+b_{1} b_{2} b_{3} \sum_{i} a_{i}+\sum_{i<j, k \neq i, j} a_{i} a_{j}\left(b_{i} b_{k}+b_{j} b_{k}\right)\right) \\
& +r_{9}\left(\sum_{i<j} a_{i} a_{j} b_{i} b_{j}+a_{1} a_{2} a_{3} \sum_{i<j} b_{i} b_{j}+b_{1} b_{2} b_{3} \sum_{i<j} a_{i} a_{j}\right) .
\end{aligned}
$$

An alternative proof can be found by writing $q^{3} \sum_{\sigma \in S_{3}} s_{\sigma 1}^{n_{1}} s_{\sigma 2}^{n_{2}} s_{\sigma 3}^{n_{3}} c\left(s_{\sigma 1}, s_{\sigma 2}, s_{\sigma 3}\right)$ as a sum $x_{0}+x_{1}(q-1)+x_{2}(q-1)^{2}+x_{3}(q-1)^{3}$, where the $x_{j}$ are rational functions of the $s_{j}$ 's, and by repeated use of $a^{k}-b^{k}=(a-b)\left(a^{k-1}+\cdots+b^{k-1}\right)$ showing that each $x_{j}$ is actually a polynomial with positive integer coefficients in the $s_{j}$ 's. 


\section{The spectrum $\Sigma$}

Each $A \in \mathscr{A}$ maps $\ell^{2}(\mathscr{V})$ into $\ell^{2}(\mathscr{V})$, and we let $\|A\|_{2,2}$ denote the corresponding operator norm. Let $\mathscr{A}_{2}$ denote the closure of $\mathscr{A}$ in the space of bounded operators on the complex Hilbert space $\ell^{2}(\mathscr{V})$ (with the usual inner product $\langle f, g\rangle=(f, \bar{g}))$. The adjoint $\left(A^{+}\right)^{\star}$ of $A^{+}$with respect to this inner product is $A^{-}$, so that $\mathscr{A}_{2}$ is a commutative $C^{*}$-algebra. In this section we find its spectrum $\Sigma$. At this point we thank Tim Steger for providing the elegant proof below, which is much shorter than our original proof, of the next important lemma, in which we estimate $\left\|A_{m, n}\right\|_{2,2}$. As we shall see in Corollary 4.6 below, this estimate is sharp.

LEMMA 4.1. Let $m, n \in \mathbb{N}$. Then $\left\|A_{m, n}\right\|_{2,2} \leq Q(m, n) / q^{m+n}$ where $Q(m, n)$ is the symmetric cubic polynomial appearing in Proposition 3.3.

PROOF. Let $\varphi_{1}$ be the spherical function defined in $(3.21)$ for $s=(1,1,1)$ (corresponding to the multiplicative functional $h_{1}$ ). Then by (3.20) and Proposition 3.3, $A_{m, n} \varphi_{1}=h_{1}\left(A_{m, n}\right) \varphi_{1}=C \varphi_{1}$ for $C=Q(m, n) / q^{m+n}$. Also, $A_{m, n}^{*} \varphi_{1}=A_{n, m} \varphi_{1}=C \varphi_{1}$ for the same $C$, because $Q(m, n)$ is symmetric. Proposition 3.3 shows that $\varphi_{1}$ is strictly positive on $\mathscr{V}$, and so the Schur Test (see [8, p. 102], for example) implies the result.

LEMMA 4.2. Let $z, w \in \mathbb{C}$ and let $\varphi_{z, w}$ be the spherical function defined in (3.21). Then $\varphi_{z, w} \notin \ell^{2}(\mathscr{V})$.

PROOF. Let $\left(s_{1}, s_{2}, s_{3}\right)$, where $s_{1} s_{2} s_{3}=1$, satisfy (3.16). Writing $p_{m, n}(z, w)=$ $q^{-m-n} p_{m, n}^{\prime}$, if $\varphi_{z, w} \in l^{2}(\mathscr{V})$, then

$$
\infty>\left\|\varphi_{z, w}\right\|_{2}^{2}=\sum_{m, n \in \mathbb{N}}\left|p_{m, n}(z, w)\right|^{2} N_{m, n} \geq \sum_{m, n \in \mathbb{N}}\left|p_{m, n}^{\prime}\right|^{2} \geq \sum_{m \in \mathbb{N}}\left|p_{m, 0}^{\prime}\right|^{2} .
$$

In particular, $p_{m, n}^{\prime}$ is bounded as $m, n \rightarrow \infty$. Let us show that this implies that $\left|s_{i}\right|=1$ for each $i$. If $s_{1}, s_{2}$ and $s_{3}$ are equal, then obviously $\left|s_{i}\right|=1$ for each $i$. If $s_{1}, s_{2}$ and $s_{3}$ are distinct, then by (3.10) $p_{m, 0}^{\prime}=\sum_{i=1}^{3} A_{i, 0} s_{i}^{m}$ can only be bounded if $\left|s_{i}\right| \leq 1$ for each $i$. For if, say, $\left|s_{1}\right| \leq\left|s_{2}\right| \leq\left|s_{3}\right|$, then (3.11) shows that $A_{3,0} \neq 0$. If, say, $s_{1} \neq s_{2}=s_{3}$, then by Proposition 3.2, we can write $p_{m, 0}^{\prime}=A_{1} s_{1}^{m}+\left(A_{2}+m A_{3}\right) s_{2}^{m}$. If $\left|s_{1}\right| \leq\left|s_{2}\right|$, then $\left|s_{2}\right| \geq 1$, we find that $A_{3} \neq 0$ and so $p_{m, 0}^{\prime}$ is unbounded. If $\left|s_{2}\right|<\left|s_{1}\right|$, then $\left|s_{1}\right|>1$ and we find that $A_{1} \neq 0$, 
and so again $p_{m, 0}^{\prime}$ is unbounded. Thus $\left|s_{i}\right| \leq 1$ for $i=1,2$. Similarly, $p_{0, n}^{\prime}$ being bounded implies that $1 /\left|s_{i}\right| \leq 1$ in each case.

Suppose then that $\left|s_{i}\right|=1$ for each $i$. Then (4.1) implies that $p_{m, 0}^{\prime} \rightarrow 0$ as $m \rightarrow \infty$. This is impossible if the $s_{i}$ are distinct, because $p_{m, 0}^{\prime} s_{1}^{-m} \rightarrow A_{1,0} \neq 0$ in the Cesàro sense. If, say, $s_{1} \neq s_{2}=s_{3}$, then $p_{m, 0}^{\prime}=A_{1} s_{1}^{m}+\left(A_{2}+m A_{3}\right) s_{2}^{m}$ is not even bounded. If the $s_{i}$ are all equal, then by Proposition 3.3, $\left|p_{m, n}^{\prime}\right|=Q(m, n)$, which is unbounded. This proves the result.

NotATion. Let us denote by $\Sigma$ the set of points $z \in \mathbb{C}$ of the form

$$
\begin{aligned}
& z=\frac{q}{q^{2}+q+1}\left(s_{1}+s_{2}+s_{3}\right) \\
& \quad \text { where }\left|s_{1}\right|=\left|s_{2}\right|=\left|s_{3}\right|=1 \text { and } s_{1} s_{2} s_{3}=1 .
\end{aligned}
$$

LEMMA 4.3. For $0<\epsilon<1$ and $z \in \Sigma$, let

$$
\varphi_{z}^{\epsilon}=\sum_{m, n \in \mathbb{N}}(1-\epsilon)^{m+n} p_{m, n}(z, \bar{z}) \chi_{m, n} .
$$

If $z$ is as in (4.2) with $s_{1}, s_{2}$ and $s_{3}$ distinct, then for each $m, n \geq 0$ there is a number $M_{m, n, z}$ such that

$$
\left\|A_{m, n} \varphi_{z}^{\epsilon}-p_{m, n}(z, \bar{z}) \varphi_{z}^{\epsilon}\right\|_{2} \leq M_{m, n, z}
$$

for each $\epsilon \in(0,1 / 2)$. As $\epsilon \rightarrow 0,\left\|\varphi_{2}^{\epsilon}\right\|_{2}^{2} \rightarrow \infty$.

PROOF. We shall prove this by induction on $m+n$, and start by proving that $\left\|A^{+} \varphi_{z}^{\epsilon}-z \varphi_{z}^{\epsilon}\right\|_{2}$ is bounded. Writing $p_{m, n}$ for $p_{m, n}(z, \bar{z})$, and using formulas (3.17), Proposition 2.3, and the fact that for $A \in \mathscr{A}$ and a biradial function

$$
\begin{aligned}
f & =\sum_{m, n} \alpha_{m, n} \chi_{m, n}, \\
A f & =\sum_{m, n} \beta_{m, n} \chi_{m, n} \quad \text { for } \quad \beta_{m, n}=\left(f, A^{*} A_{n, m} \delta_{o}\right),
\end{aligned}
$$

we find that

$$
\begin{aligned}
A^{+} \varphi_{z}^{\epsilon}-z \varphi_{z}^{\epsilon}=-\epsilon z \chi_{0,0} & -\frac{\epsilon}{q^{2}+q+1} \sum_{n=1}^{\infty}\left(\left(q^{2}+q\right) p_{1, n}-\frac{1}{1-\epsilon} p_{0, n-1}\right)(1-\epsilon)^{n} \chi_{0, n} \\
& -\frac{\epsilon}{q^{2}+q+1} \sum_{m=1}^{\infty} q^{2} p_{m+1,0}(1-\epsilon)^{m} \chi_{m, 0} \\
& -\frac{\epsilon}{q^{2}+q+1} \sum_{m, n \geq 1}\left(q^{2} p_{m+1, n}-\frac{1}{1-\epsilon} p_{m, n-1}\right)(1-\epsilon)^{m+n} \chi_{m, n} .
\end{aligned}
$$


From (3.15) we see that $\left|p_{m, n}\right| \leq C_{z} / q^{m+n}$ for a number $C_{z}$ dependingtonly on $z$. Thus

$$
\begin{aligned}
\left\|A^{+} \varphi_{z}^{\epsilon}-z \varphi_{2}^{\epsilon}\right\|_{2}^{2} \leq \epsilon^{2} C_{z}^{2}\left(M_{1}\right. & +M_{2} \sum_{n=1}^{\infty}(1-\epsilon)^{2 n} \\
& \left.+M_{3} \sum_{m=1}^{\infty}(1-\epsilon)^{2 m}+M_{4} \sum_{m, n=1}^{\infty}(1-\epsilon)^{2(m+n)}\right)
\end{aligned}
$$

for numbers $M_{1}, M_{2}, M_{3}$ and $M_{4}$ depending only on $q$. It follows that $\| A^{+} \varphi_{z}^{\epsilon}-$ $z \varphi_{z}^{\epsilon} \|_{2}$ is bounded. One can show that $\left\|A^{-} \varphi_{z}^{\epsilon}-\bar{z} \varphi_{z}^{\epsilon}\right\|_{2}$ is bounded similarly or by using $\left(A^{+}\right)^{*}=A^{-},\left(\varphi_{z, \bar{z}}^{\epsilon}\right)^{*}=\varphi_{\bar{z}, z}^{\epsilon}$, Formula (2.2), and what we have just proved. The rest of the proof of 4.3 is a routine induction on $m+n$, using (3.1)-(3.7) and the formulas in Proposition 2.3. The last statement follows immediately from Lemma 4.2.

PROPOSITION 4.4. Let $z, w \in \mathbb{C}$. The multiplicative functional $h_{z, w}$ on $\mathscr{A}$ is continuous for the $\ell^{2}$ operator norm on $\mathscr{A}$ if and only if $w=\vec{z}$ and $z \in \Sigma$. The map $z \mapsto h_{z, \bar{z}}$ is thus a homeomorphism of $\Sigma$ onto the spectrum of the $C^{*}$-algebra $\mathscr{A}_{2}$.

PROOF. Suppose that $h_{z, w}$ is continuous for the norm $\|A\|_{2,2}$. Then $\left|h_{z, w}\left(A_{m, n}\right)\right|$ $\leq\left\|A_{m, n}\right\|_{2,2}$ for each $m, n \geq 0$. Hence, by Lemma 4.1, $\left|p_{m, n}(z, w)\right| \leq$ $Q(m, n) / q^{m+n}$. Let $\left(s_{1}, s_{2}, s_{3}\right) \in \mathbb{C}^{3}$, satisfying $s_{1} s_{2} s_{3}=1$, be related to $(z, w)$ as in (3.16). Writing $p_{m, n}^{\prime}=q^{m+n} p_{m, n}(z, w)$, the condition $\left|p_{m, n}^{\prime}\right| \leq Q(m, n)$ for each $m, n \geq 0$ implies, as in the proof of Lemma 4.2, that $\left|s_{i}\right|=1$ for each $i$, so that $w=\bar{z}$ and $z \in \Sigma$. Of course, $w=\bar{z}$ is also an immediate consequence of $A^{-}=\left(A^{+}\right)^{\star}$ and the assumed continuity of $h_{z, w}$.

Conversely, suppose that $z \in \Sigma$ is as in (4.2) with the $s_{j}$ 's distinct. Then by Lemma 4.3,

$$
\frac{\left\|A^{+} \varphi_{z}^{\epsilon}-z \varphi_{z}^{\epsilon}\right\|_{2}}{\left\|\varphi_{z}^{\epsilon}\right\|_{2}} \rightarrow 0
$$

which implies that $z$ is in the spectrum $\sigma\left(A^{+}\right)$of $A^{+}$on $\ell^{2}(\mathscr{V})$. As this spectrum is closed, and coincides with the set of values $h\left(A^{+}\right)$, where $h$ is a continuous multiplicative functional, the result follows.

Let us now describe the set $\Sigma$ explicitly. 
PROPOSITION 4.5. The set $\Sigma$ is a hypocycloid with three cusps and its interior (see Figure 4). It is the set of points $z \in \mathbb{C}$ for which

$4 q\left(q^{2}+q+1\right)^{3}\left(z^{3}+\bar{z}^{3}\right)-\left(q^{2}+q+1\right)^{4} z^{2} \bar{z}^{2}-18 q^{2}\left(q^{2}+q+1\right)^{2} z \bar{z}+27 q^{4} \geq 0$.

PROOF. Firstly, $\Sigma$ is the set $\Sigma_{0}$, dilated by the factor $q /\left(q^{2}+q+1\right)$, where

$$
\Sigma_{0}=\left\{s_{1}+s_{2}+s_{3}:\left|s_{1}\right|=\left|s_{2}\right|=\left|s_{3}\right|=1 \text { and } s_{1} s_{2} s_{3}=1\right\} .
$$

The Jacobian of the transformation

$$
\begin{aligned}
& x=\cos \left(\theta_{1}\right)+\cos \left(\theta_{2}\right)+\cos \left(\theta_{1}+\theta_{2}\right) \\
& y=\sin \left(\theta_{1}\right)+\sin \left(\theta_{2}\right)-\sin \left(\theta_{1}+\theta_{2}\right)
\end{aligned}
$$

is, writing $\theta_{3}$ for $-\theta_{1}-\theta_{2}$,

$$
J=\frac{1}{2 i}\left(e^{i \theta_{1}}-e^{i \theta_{2}}\right)\left(e^{i \theta_{2}}-e^{i \theta_{3}}\right)\left(e^{i \theta_{1}}-e^{i \theta_{3}}\right) .
$$

Thus

$$
|J|^{2}=4\left(z^{3}+\bar{z}^{3}\right)-z^{2} \bar{z}^{2}-18 z \bar{z}+27 \quad \text { if } \quad z=e^{i \theta_{1}}+e^{i \theta_{2}}+e^{i \theta_{3}}
$$

(see Formula (5.5) below). The boundary of $\Sigma_{0}$ is given by the vanishing of $J$, and so is the set of points $s_{1}+s_{2}+s_{3}$, where $\left|s_{1}\right|=\left|s_{2}\right|=\left|s_{3}\right|=1, s_{1} s_{2} s_{3}=1$, and the $s_{i}$ 's are not distinct. But if $s_{i}=s_{j}$, with $i \neq j$, we get a hypocycloid.

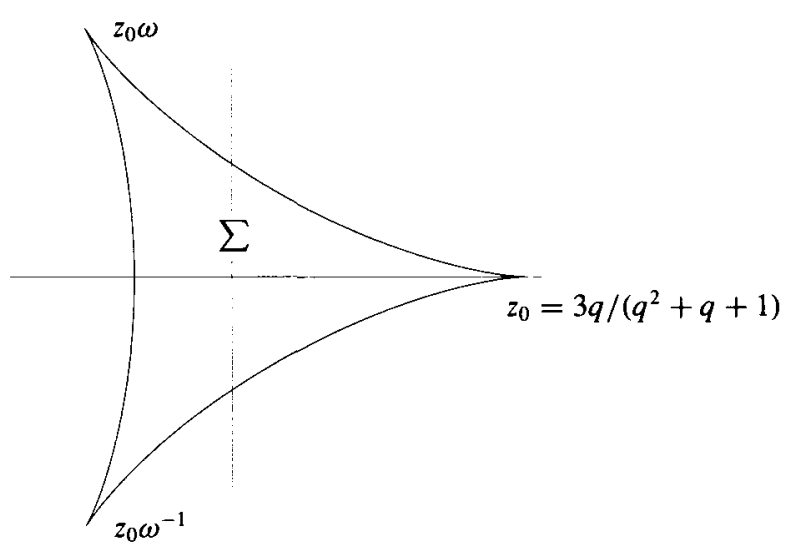

FIGURE 4 
COROLlARY 4.6. For each $m, n \geq 0$ we have, in the notation of Proposition 3.3, $\left\|A_{m, n}\right\|_{2,2}=Q(m, n) / q^{m+n}$.

PROOF. By Proposition 4.4, the multiplicative functional $h_{1}$ corresponding to $s=(1,1,1)$ is continuous for the $\ell^{2}$ operator norm on $\mathscr{A}$. Thus $\left\|A_{m, n}\right\|_{2,2} \geq$ $\left|h_{1}\left(A_{m, n}\right)\right|=Q(m, n) / q^{m+n}$. The reverse inequality is just Lemma 4.1.

REMARKS. Each $A \in \mathscr{A}$ also maps $\ell^{1}(\mathscr{V})$ into $\ell^{1}(\mathscr{V})$. If $\|A\|_{1,1}$ is the corresponding operator norm and if $\mathscr{A}_{1}$ is the closure of $\mathscr{A}$ in the space of bounded operators on $\ell^{1}(\mathscr{V})$, we can also calculate the spectrum of this Banach algebra. Indeed, let $z, w \in \mathbb{C}$ and let $s=\left(s_{1}, s_{2}, s_{3}\right) \in S$ be related as in (3.16). Then $h_{z, w}$ is continuous for the norm $\|A\|_{1,1}$ if and only if the spherical function $\varphi_{z, w}=\varphi_{s}$ is bounded, and this holds if and only if

$$
\frac{1}{q} \leq\left|s_{i}\right| \leq q \quad(i=1,2,3) .
$$

The first statement holds because $\left\|A_{m, n}\right\|_{1,1}=1$ for each $m, n$, and the second is proved as in Lemma 4.2. Condition (4.4) may be expressed geometrically: it holds if and only if $\left(\log \left|s_{1}\right|, \log \left|s_{2}\right|, \log \left|s_{3}\right|\right)$ lies in the hexagon whose six vertices are $(\log q, 0,-\log q)$ and its permutations. Thus the above statement is essentially a very special case of [7, Theorem 4.7.1].

PROPOSITION 4.7. Let $z \in \Sigma$. Define $k_{z}: \mathscr{V} \times \mathscr{V} \rightarrow \mathbb{C}$ by $k_{z}(u, v)=$ $p_{m, n}(z, \bar{z})$ if $v \in S_{m, n}(u)$. Then $k_{z}$ is a positive definite kernel, that is, for any integer $n \geq 1$ and any $v_{1}, \ldots, v_{n} \in \mathscr{V}$, the matrix $(j, k) \mapsto k_{z}\left(v_{j}, v_{k}\right)$ is positive definite. If $g \in \operatorname{Aut}_{\mathrm{tr}}(\Delta)$, then $k_{z}(g u, g v)=k_{z}(u, v)$, and so $g \mapsto \varphi_{z, \bar{z}}(g o)=$ $k_{z}(o, g o)$ is positive definite on $\operatorname{Aut}_{\mathrm{tr}}(\Delta)$.

PROOF. Let $z$ be given by (4.2), where $s_{1}, s_{2}$ and $s_{3}$ are distinct. Observe that $k_{z}(u, v)=\left(\varphi_{z, \bar{z}}^{u}\right)(v)$, where, for any (o-)biradial function $f$, we define the $u$-biradial function $f^{u}$ by

$$
f^{u}=\sum_{m, n} \alpha_{m, n} \chi_{S_{m, n}(u)} \quad \text { if } \quad f=\sum_{m, n} \alpha_{m, n} \chi_{S_{m, n}(o)} .
$$

Notice that $\left\langle f^{u}, g^{u}\right\rangle=\langle f, g\rangle$ for biradial $f, g \in \ell^{2}(\mathscr{V})$. If $\mathscr{E}^{v}$ is the natural projection onto the space of $v$-biradial functions, we have, for any $o$-biradial function $f$,

$$
\mathscr{E}^{v} f^{u}=A_{m, n} f^{v} \quad \text { if } v \in S_{m, n}(u) .
$$


Indeed, if $f=A_{r, s} \delta_{o}=\chi_{s_{s, r}(o)} / N_{r, s}$, then $f^{u}=\chi_{s_{s, r}(u)} / N_{r, s}=A_{r, s} \delta_{u}$. Because $\mathscr{E}^{v}$, like $\mathscr{E}$, commutes with each $A \in \mathscr{A}$, we have

$$
\mathscr{E}^{v} f^{u}=\mathscr{E}^{v} A_{r, s} \delta_{u}=A_{r, s} \mathscr{E}^{v} \delta_{u}=A_{r, s} \chi_{S_{n, m}(v)} / N_{m, n}=A_{r, s} A_{m, n} \delta_{v}=A_{m, n} f^{v}
$$

If $u \mapsto f_{u}$ is any map $\mathscr{V} \rightarrow \ell^{2}(\mathscr{V}),(u, v) \mapsto\left\langle f_{u}, f_{v}\right\rangle$ is a positive definite kernel. So it is sufficient to prove that, in the notation of Lemma 4.3,

$$
k_{z}(u, v)=\lim _{\epsilon \rightarrow 0}\left\langle\left(\varphi_{z}^{\epsilon}\right)^{u},\left(\varphi_{z}^{\epsilon}\right)^{v}\right\rangle /\left\|\varphi_{z}^{\epsilon}\right\|_{2}^{2} .
$$

But if $v \in S_{m, n}(u)$,

$$
\begin{aligned}
\left\langle\left(\varphi_{z}^{\epsilon}\right)^{u},\left(\varphi_{z}^{\epsilon}\right)^{v}\right\rangle-k_{z}(u, v)\left\|\varphi_{z}^{\epsilon}\right\|_{2}^{2} & =\left\langle\left(\varphi_{z}^{\epsilon}\right)^{u}-p_{m, n}(z, \bar{z})\left(\varphi_{z}^{\epsilon}\right)^{v},\left(\varphi_{z}^{\epsilon}\right)^{v}\right\rangle \\
& =\left\langle\mathscr{E}^{v}\left(\left(\varphi_{z}^{\epsilon}\right)^{u}\right)-p_{m, n}(z, \bar{z})\left(\varphi_{z}^{\epsilon}\right)^{v},\left(\varphi_{z}^{\epsilon}\right)^{v}\right\rangle \\
& =\left\langle A_{m, n}\left(\left(\varphi_{z}^{\epsilon}\right)^{v}\right)-p_{m, n}(z, \bar{z})\left(\varphi_{z}^{\epsilon}\right)^{v},\left(\varphi_{z}^{\epsilon}\right)^{v}\right\rangle
\end{aligned}
$$

and, by Lemma 4.3, in modulus this is at most $M_{m, n, z}\left\|\varphi_{z}^{\epsilon}\right\|_{2}$.

If $z$ is given by (4.2) but the $s_{i}$ are not distinct, $k_{z}$ is still positive definite, being the pointwise limit of $k_{z_{j}}$ for a sequence $\left(z_{j}\right)$ of 'nonsingular' $z_{j} \in \Sigma$.

\section{The spherical Plancherel formula}

Let $A \mapsto \hat{A}$, where $\hat{A}(z)=h_{z, \bar{z}}(A)$, be the Gelfand isomorphism. The Plancherel measure $\mu$ on $\Sigma$ is determined by the condition that

$$
\int_{\Sigma} \widehat{A}_{m, n}(z) d \mu(z)= \begin{cases}1 & \text { if } m=n=0 \\ 0 & \text { otherwise }\end{cases}
$$

The following theorem is essentially a special case of [7, Theorem 5.1.2]:

PROPOSITION 5.1. Let $S_{1}=\left\{\left(s_{1}, s_{2}, s_{3}\right) \in \mathbb{T}^{3}: s_{1} s_{2} s_{3}=1\right\}$, let ds denote normalized Haar measure on $S_{1}$, and let $\psi: S_{1} \rightarrow \Sigma$ be the map $\left(s_{1}, s_{2}, s_{3}\right) \mapsto$ $q\left(s_{1}+s_{2}+s_{3}\right) /\left(q^{2}+q+1\right)$. Then the Plancherel measure $\mu$ is the image under $\psi$ of the following measure on $S_{1}$ :

$$
\frac{(q+1)\left(q^{2}+q+1\right)}{6 q^{3}} \frac{1}{|c(s)|^{2}} d s .
$$


PROOF. (cf. [7, pp. 65-67]) We verify that (5.1) holds for the measure $\mu$ on $\Sigma$ which is the image under $\psi$ of the measure on $S_{1}$ given by (5.2). Using Proposition 3.1, continuity of $\widehat{A}_{m, n}(z)$, and the fact that for $s=\left(s_{1}, s_{2}, s_{3}\right) \in S_{1}$,

$$
\left|c\left(s_{\sigma 1}, s_{\sigma 2}, s_{\sigma 3}\right)\right|^{2}=c\left(s_{\sigma 1}, s_{\sigma 2}, s_{\sigma 3}\right) c\left(\bar{s}_{\sigma 1}, \bar{s}_{\sigma 2}, \bar{s}_{\sigma 3}\right)
$$

does not depend on the permutation $\sigma$, we have, writing $M=(q+1)\left(q^{2}+q+\right.$ 1) $/ 6 q^{3}$

$$
\begin{aligned}
\int_{\Sigma} \widehat{A}_{m, n}(z) d \mu(z) & =\int_{S_{1}} \widehat{A}_{m, n}(\psi(s)) \frac{M}{|c(s)|^{2}} d s \\
& =\frac{1}{6 q^{m+n}} \sum_{\sigma \in S_{3}} \int_{S_{1}} \frac{s_{\sigma 1}^{n_{1}} s_{\sigma 2}^{n_{2}} s_{\sigma 3}^{n_{3}}}{c\left(\bar{s}_{\sigma 1}, \bar{s}_{\sigma 2}, \bar{s}_{\sigma 3}\right)} d s
\end{aligned}
$$

where $n_{1} \geq n_{2} \geq n_{3}$ are integers, with $m=n_{1}-n_{2}$ and $n=n_{2}-n_{3}$. Now

$$
\frac{1}{c\left(\bar{s}_{1}, \bar{s}_{2}, \bar{s}_{3}\right)}=\prod_{i<j} \frac{\bar{s}_{i}-\bar{s}_{j}}{\bar{s}_{i}-\frac{\bar{s}_{j}}{q}}=\prod_{i<j}\left(1-(q-1) \sum_{k=1}^{\infty}\left(\frac{s_{i}}{q s_{j}}\right)^{k}\right) \text {. }
$$

So replacing $\left(s_{1}, s_{2}, s_{3}\right)$ by $\left(s_{\sigma 1}, s_{\sigma 2}, s_{\sigma 3}\right)$ and multiplying out the product, the last integrand in (5.3) may be expanded in an absolutely and uniformly convergent series of integrals

$$
\int_{S_{1}} s_{\sigma 1}^{n_{1}+k_{1}+k_{2}} s_{\sigma 2}^{n_{2}+k_{3}-k_{1}} s_{\sigma 3}^{n_{3}-k_{2}-k_{3}} d s
$$

where $k_{1}, k_{2}, k_{3} \geq 0$. Remembering that $n_{1} \geq n_{2} \geq n_{3}$, we see that the three exponents $n_{1}+k_{1}+k_{2}, n_{2}+k_{3}-k_{1}$ and $n_{3}-k_{2}-k_{3}$ cannot be equal unless $k_{1}=k_{2}=k_{3}=0$ and $n_{1}=n_{2}=n_{3}$. Thus all the integrals (5.4) are zero unless $k_{1}=k_{2}=k_{3}=0$ and $n_{1}=n_{2}=n_{3}$, so that $\int_{\Sigma} \widehat{A_{m, n}}(z) d \mu(z)=0$ unless $m=n=0$, in which case this integral reduces to

$$
\frac{1}{6} \sum_{\sigma \in S_{3}} \int_{S_{1}} s_{\sigma 1}^{n_{1}} s_{\sigma 2}^{n_{1}} s_{\sigma 3}^{n_{1}} d s=1 .
$$

This completes the proof.

Let us now work out a more explicit formula for the measure $\mu$. Using the identity

$$
\begin{aligned}
& \left|\left(q s_{1}-s_{2}\right)\left(q s_{1}-s_{3}\right)\left(q s_{2}-s_{3}\right)\right|^{2} \\
& \quad=q^{2}(q+1)^{2}\left(z_{1}^{3}+\bar{z}_{1}^{3}\right)-q^{3} z_{1}^{2} \bar{z}_{1}^{2}-q\left(q^{2}+q+1\right)\left(q^{2}+4 q+1\right) z_{1} \bar{z}_{1}+\left(q^{2}+q+1\right)^{3}
\end{aligned}
$$


for $\left(s_{1}, s_{2}, s_{3}\right) \in S_{1}$ and $z_{1}=s_{1}+s_{2}+s_{3}$, we find that if $z=\psi(s) \in \Sigma$, then

$$
\begin{aligned}
& \frac{1}{|c(s)|^{2}} \\
& =\frac{q^{3}}{\left(q^{2}+q+1\right)^{3}} \\
& \times\left\{\frac{4 q\left(q^{2}+q+1\right)^{3}\left(z^{3}+\bar{z}^{3}\right)-\left(q^{2}+q+1\right)^{4} z^{2} \bar{z}^{2}-18 q^{2}\left(q^{2}+q+1\right)^{2} z \bar{z}+27 q^{4}}{(q+1)^{2}\left(z^{3}+\bar{z}^{3}\right)-\left(q^{2}+q+1\right) z^{2} \bar{z}^{2}-\left(q^{2}+4 q+1\right) z \bar{z}+q}\right\} \\
& =\frac{q^{3}}{\left(q^{2}+q+1\right)^{3}} \alpha(\psi(s)), \quad \text { say. }
\end{aligned}
$$

Thus $\int_{\Sigma} f(z) d \mu(z)=M_{1} \int_{\Sigma} g(z) d \mu^{\prime}(z)$, where $M_{1}=(q+1) / 6\left(q^{2}+q+1\right)^{2}$, $g(z)=f(z) \alpha(z)$, and $\mu^{\prime}$ is the image under $\psi$ of the normalized Haar measure on $S_{1}$. Now

$$
\begin{aligned}
\int_{\Sigma} g(z) d \mu^{\prime}(z) & =\int_{S_{1}} g(\psi(s)) d s \\
& =\frac{1}{4 \pi^{2}} \int_{-\pi}^{\pi} \int_{-\pi}^{\pi} g\left(\psi\left(e^{i \theta_{1}}, e^{i \theta_{2}}, e^{-i\left(\theta_{1}+\theta_{2}\right)}\right)\right) d \theta_{1} d \theta_{2} .
\end{aligned}
$$

The Jacobian of the transformation

$$
\begin{aligned}
& x=\frac{q}{q^{2}+q+1}\left(\cos \left(\theta_{1}\right)+\cos \left(\theta_{2}\right)+\cos \left(\theta_{1}+\theta_{2}\right)\right) \\
& y=\frac{q}{q^{2}+q+1}\left(\sin \left(\theta_{1}\right)+\sin \left(\theta_{2}\right)-\sin \left(\theta_{1}+\theta_{2}\right)\right)
\end{aligned}
$$

is, writing $\theta_{3}$ for $-\theta_{1}-\theta_{2}$,

$$
J=\frac{q^{2}}{\left(q^{2}+q+1\right)^{2}} \frac{1}{2 i}\left(e^{i \theta_{1}}-e^{i \theta_{2}}\right)\left(e^{i \theta_{2}}-e^{i \theta_{3}}\right)\left(e^{i \theta_{1}}-e^{i \theta_{3}}\right) .
$$

Thus, by (5.5) again,

$$
\begin{aligned}
& J^{2}=|J|^{2} \\
& =\frac{4 q\left(q^{2}+q+1\right)^{3}\left(z^{3}+\bar{z}^{3}\right)-\left(q^{2}+q+1\right)^{4} z^{2} \bar{z}^{2}-18 q^{2}\left(q^{2}+q+1\right)^{2} z \bar{z}+27 q^{4}}{4\left(q^{2}+q+1\right)^{4}} .
\end{aligned}
$$

Applying the change of variable formula, we have

$$
\int_{\Sigma} g(z) d \mu^{\prime}(z)=\frac{\left(q^{2}+q+1\right)^{2}}{2 \pi^{2}} \iint_{\Sigma} g(z) r(z) d x d y
$$


for

$r(z)=\frac{1}{\sqrt{4 q\left(q^{2}+q+1\right)^{3}\left(z^{3}+\bar{z}^{3}\right)-\left(q^{2}+q+1\right)^{4} z^{2} \bar{z}^{2}-18 q^{2}\left(q^{2}+q+1\right)^{2} z \bar{z}+27 q^{4}}}$.

Combining the above calculations, we have therefore proved:

PROPOSITION 5.2. The Plancherel measure on $\Sigma$ is given by

$$
\int_{\Sigma} f(z) d \mu(z)=\frac{q+1}{12 \pi^{2}} \iint_{\Sigma} f(z) R(z) d x d y
$$

where $d x$ dy denotes Lebesgue measure on $\mathbb{R}^{2}=\mathbb{C}$, and where

$$
R(z)=\frac{\sqrt{4 q\left(q^{2}+q+1\right)^{3}\left(z^{3}+\bar{z}^{3}\right)-\left(q^{2}+q+1\right)^{4} z^{2} \bar{z}^{2}-18 q^{2}\left(q^{2}+q+1\right)^{2} z \bar{z}+27 q^{4}}}{(q+1)^{2}\left(z^{3}+\bar{z}^{3}\right)-\left(q^{2}+q+1\right) z^{2} \bar{z}^{2}-\left(q^{2}+4 q+1\right) z \bar{z}+q}
$$

Now suppose that $G$ is a closed subgroup of $\operatorname{Aut}_{\mathrm{tr}}(\Delta)$. Let $\lambda$ be the regular representation of $G$ on $\ell^{2}(\mathscr{V}): \lambda(g) f(u)=f\left(g^{-1} u\right)$. Then we obtain direct integral decompositions $\ell^{2}(\mathscr{V})=\oplus \int_{\Sigma} \mathscr{H}_{z} d \mu(z)$ and $\lambda=\oplus \int_{\Sigma} \pi_{z} d \mu(z)$ in the usual way. We omit the details, referring the reader to [6] or [9], for example, except to say that one starts by defining $\left\langle f_{1}, f_{2}\right\rangle_{z}=\sum_{u, v \in \mathscr{V}} f_{1}(u) \overline{f_{2}(v)} k_{z}(u, v)$ for finitely supported $f_{1}, f_{2}$ on $\mathscr{V}$, with $k_{z}$ as in Proposition 4.7 .

\section{EXAMPLES.}

(a) Suppose that $G$ acts transitively on $\mathscr{V}$, and that $K=\{g \in G: g o=o\}$ acts transitively on each $S_{m, n}(o)$. Then $(G, K)$ is a Gelfand pair (Proposition 2.4) and so the representations $\pi_{z}$ are irreducible and pairwise inequivalent (see, for example, [5, Chapter II, Theorem 5.3]). For example, this holds when $\Delta=\Delta_{F}$ and $G=P G L(3, F)$.

(b) Let $\Delta=\Delta_{\mathscr{T}}$, and suppose that $\Gamma_{\mathscr{T}}$ embeds as a lattice in $\operatorname{PGL}(3, F)$ for some $F$. Then by a recent theorem of Cowling and Steger [4], the $\pi_{z}$ remain irreducible and pairwise inequivalent.

(c) Let $\Delta=\Delta_{\mathscr{T}}$, and suppose that $\Gamma_{\mathscr{T}}$ does not embed as a lattice in $\operatorname{PGL}(3, F)$ for any $F$. Examples of such $\mathscr{T}$ appear in [3]. Then all we can currently say is that almost all of the $\pi_{z}$ are irreducible. This follows from the fact that the weak closure of $\mathscr{A}$ in the von Neumann algebra $\mathscr{L}$ generated on $\ell^{2}\left(\Gamma_{\mathscr{T}}\right)$ by the left regular representation is a maximal abelian subalgebra of $\mathscr{L}$ (see [9]). This in turn follows from Theorem 6.1 in the next section. 


\section{The maximal abelian property}

In this section we consider the case $\Delta=\Delta_{\mathscr{T}}$ (see Section 1), in which $\mathscr{A}$ may be identified with the convolution algebra on $\Gamma_{\mathscr{T}}$ generated by $\mu^{+}$and $\mu^{-}$ (see Section 2). Our aim, for reasons explained at the end of Section 5, is to prove the following result:

THEOREM 6.1. Let $f \in \ell^{2}\left(\Gamma_{\mathscr{T}}\right)$, and suppose that $f * \mu^{+}=\mu^{+} * f$ and $f * \mu^{-}=\mu^{-} * f$. Then $f$ is biradial (with respect to the identity element 1 ).

Notation. For $x, y \in P$, we shall simply write $x$ and $y^{-1}$ for the elements $a_{x}$ and $a_{y}^{-1}$, respectively, of $\Gamma_{\mathscr{T}}$. We know from Proposition 3.2 in [2] that each $g \in \Gamma_{\mathscr{T}}$ can be written uniquely in the form

$$
g=x_{1} x_{2} \cdots x_{m} y_{1}^{-1} \cdots y_{n}^{-1}
$$

where the $x_{i}$ and $y_{j}$ are in $P$, and there is no 'obvious' way of shortening this word for $g$ :

(a) $m, n \geq 0$ are integers;

(b) $x_{i+1} \notin \lambda\left(x_{i}\right)$ for $1 \leq i<m$;

(c) $y_{j} \notin \lambda\left(y_{j+1}\right)$ for $1 \leq j<n$;

(d) $x_{m} \neq y_{1}$ (if $m, n \geq 1$ ).

We shall call this the right normal form of $g$, and refer to the number $m+n$ as the length of $g$, and denote it $l(g)$. This word for $g$ has the geometric interpretation of the shortest path $1=u \rightarrow v^{\prime} \rightarrow v=g$ between 1 and $g$ (see Figure 1).

We need to know that each element can also be written uniquely with the inverse generators on the left and no obvious cancellations:

LEMMA 6.2. Each $g \in \Gamma_{\mathscr{T}}$ can be written uniquely in the form

$$
g=v_{1}^{-1} \cdots v_{n^{\prime}}^{-1} u_{1} u_{2} \cdots u_{m^{\prime}}
$$

where

(a) $m^{\prime}, n^{\prime} \geq 0$ are integers;

(b) $u_{i+1} \notin \lambda\left(u_{i}\right)$ for $1 \leq i<m^{\prime}$;

(c) $v_{j} \notin \lambda\left(v_{j+1}\right)$ for $1 \leq j<n^{\prime}$;

(d) $v_{n^{\prime}} \neq u_{1}\left(\right.$ if $\left.m^{\prime}, n^{\prime} \geq 1\right)$. 
Moreover, if $g$ is as in (6.1), then $m^{\prime}=m$ and $n^{\prime}=n$. We call (6.2) the left normal form of $g$. Any minimal word for $g$ in the $x$ 's and their inverses contains $m x$ 's and $n y^{-1}$ 's.

PROOF. To show the existence of a left normal form for $g$, take any minimal word for $g$ in the $x$ 's and $y^{-1}$ 's. If $u, v \in P$ and $u \neq v$, there is a unique $s \in P$ such that $s \in \lambda(u)$ and $s \in \lambda(v)$. Then there are unique $x, y \in P$ such that $(u, s, x) \in \mathscr{T}$ and $(v, s, y) \in \mathscr{T}$. Thus $a_{u} a_{s} a_{x}=1=a_{v} a_{s} a_{y}$. So $a_{x}^{-1} a_{y}=a_{u} a_{v}^{-1}$, or in the more concise notation, $u v^{-1}=x^{-1} y$. In this way, inverse generators can be moved from right to left, and after a finite number of steps we obtain a word for $g$ in left normal form.

If the uniqueness were false, we could pick a $g \in \Gamma$ having two distinct left normal forms:

$$
g=v_{1}^{-1} \cdots v_{n^{\prime}}^{-1} u_{1} \cdots u_{m^{\prime}} \quad \text { and } \quad g=l_{1}^{-1} \cdots l_{s}^{-1} k_{1} \cdots k_{r}
$$

with $m^{\prime}+n^{\prime}$ minimal. Thus

$$
l_{s} \cdots l_{1} v_{1}^{-1} \cdots v_{n^{\prime}}^{-1}=k_{1} \cdots k_{r} u_{m^{\prime}}^{-1} \cdots u_{1}^{-1} .
$$

By minimality, if $s, n^{\prime} \geq 1$, then $l_{1} \neq v_{1}$, and if $r, m^{\prime} \geq 1$, then $k_{r} \neq u_{m^{\prime}}$. So by the uniqueness of right normal forms, the words $l_{s} \cdots l_{1} v_{1}^{-1} \cdots v_{n^{\prime}}^{-1}$ and $k_{1} \cdots k_{r} u_{m^{\prime}}^{-1} \cdots u_{1}^{-1}$ must be identical. But this contradicts the hypothesis that the two words for $g$ in (6.3) are in left normal form.

To see that $m^{\prime}=m$ and $n^{\prime}=n$, just observe that, starting from any minimal word for $g$, moving inverse generators from right to left as above to obtain a left normal form, or moving them from left to right to get a right normal form, we don't change the number of inverse generators present.

Write $S_{j, k}$ for $S_{j, k}(1)$, where 1 is the identity element of $\Gamma_{\mathscr{T}}$. It is not hard to prove by induction that $S_{j, k}$ is just the set of elements $g \in \Gamma_{\mathscr{T}}$ for which $m=j$ and $n=k$ is the last lemma (one uses the fact that $\Gamma_{\mathscr{T}}$ embeds in $\operatorname{Aut}_{\mathrm{tr}}\left(\Delta_{\mathscr{T}}\right.$ ) by left multiplication).

Let $w \in S_{r, s}$, where $r, s \geq 0$. Write

$$
w=i_{r} \cdots i_{1} j_{1}^{-1} \cdots j_{s}^{-1}=l_{s}^{-1} \cdots l_{1}^{-1} k_{1} \cdots k_{r}
$$

(the right and left normal forms of $w$ ). We can re-interpret Lemma 2.1 in the current context as follows. For example, using the fact that $v \in S_{j, k}(u)$ if and only if $v=u \xi$ for some $\xi \in S_{j, k}$, the second part of Lemma 2.1(a) becomes:

Let $w \in S_{r, s}$ be as in (6.4), where $r, s \geq 1$. Let $x \in P$. Then one of the following three mutually exclusive possibilities occurs: 
(a) $x=l_{s}$; then $l(x w)=l(w)-1$ and $x w \in S_{r, s-1}$.

(b) $x \neq l_{s}$ but $i_{r} \in \lambda(x)$; then $l(x w)=l(w)$ and $x w \in S_{r-1, s+1}$. This holds for exactly $q x$ 's.

(c) $i_{r} \notin \lambda(x)$; then $l(x w)=l(w)+1$ and $x w \in S_{r+1, s}$. This holds for exactly $q^{2} x$ 's.

NotATION. It is convenient to work in the group algebra $\mathbb{C}\left(\Gamma_{\mathscr{T}}\right)$ of $\Gamma_{\mathscr{T}}$, and to use the non-normalized convolution operators $f \mapsto \chi^{+} * f$ and $f \mapsto \chi^{-} * f$, where

$$
\chi^{+}=\sum_{x \in P} x \in \mathbb{C}\left(\Gamma_{\mathscr{T}}\right) \quad \text { and } \quad \chi^{-}=\sum_{y \in P} y^{-1} \in \mathbb{C}\left(\Gamma_{\mathscr{T}}\right)
$$

Let $w \in S_{r, s}$, where $r, s \geq 0$. With notation as in (6.4), if $s \geq 1$, write

$$
w^{\prime}=l_{s-1}^{-1} \cdots l_{1}^{-1} k_{1} \cdots k_{r} \quad \text { and } \quad w^{\prime \prime}=i_{r} \cdots i_{1} j_{1}^{-1} \cdots j_{s-1}^{-1} \quad\left(\in S_{r, s-1}\right)
$$

and if $r \geq 1$, write

$$
w^{*}=i_{r-1} \cdots i_{1} j_{1}^{-1} \cdots j_{s}^{-1} \quad \text { and } \quad w^{* *}=l_{s}^{-1} \cdots l_{1}^{-1} k_{1} \cdots k_{r-1} \quad\left(\in S_{r-1, s}\right)
$$

LEMMA 6.3. Let $w \in S_{r, s}$, as in (6.4), where $r \geq 0$ and $s \geq 1$. Let $f_{0}=w \in$ $\mathbb{C}\left(\Gamma_{\mathscr{T}}\right)$, and for $n \geq 1$ let

$$
\begin{aligned}
f_{n}=\frac{1}{2 q^{2 n}}\left\{\sum x_{n}\right. & \cdots x_{1} i_{r} \cdots i_{1} j_{1}^{-1} \cdots j_{s}^{-1} v_{1}^{-1} \cdots v_{n}^{-1} \\
& \left.+\sum u_{n}^{-1} \cdots u_{1}^{-1} l_{s}^{-1} \cdots l_{1}^{-1} k_{1} \cdots k_{r} y_{1} \cdots y_{n}\right\}
\end{aligned}
$$

and

$$
\begin{aligned}
g_{n}=\frac{-1}{2 q^{2 n-1}}\left\{\sum x_{n-1} \cdots x_{1} i_{r} \cdots i_{1} j_{1}^{-1} \cdots j_{s}^{-1} v_{1}^{-1} \cdots v_{n}^{-1}\right. \\
\\
\left.+\sum u_{n}^{-1} \cdots u_{1}^{-1} l_{s}^{-1} \cdots l_{1}^{-1} k_{1} \cdots k_{r} y_{1} \cdots y_{n-1}\right\}
\end{aligned}
$$


where the sums are over the $x_{i}$ 's, $y_{i}$ 's, $u_{i}$ 's and $v_{i}$ 's in $P$ for which all the terms in the sums are in normal form, as written. Then for $n \geq 1$ we can write

$$
\begin{array}{r}
\left(\chi^{+}\left(f_{0}+\cdots+f_{n}\right)-\left(f_{0}+\cdots+f_{n}\right) \chi^{+}\right)+\left(\chi^{-}\left(g_{1}+\cdots+g_{n}\right)-\left(g_{1}+\cdots+g_{n}\right) \chi^{-}\right) \\
=w^{\prime}-w^{\prime \prime}+f_{r}+\frac{1}{2 q^{2 n-1}}\left(A_{n}-B_{n}\right)+\frac{1}{2 q^{2 n}}\left(C_{n}-D_{n}+E_{n}-F_{n}\right)
\end{array}
$$

where $f_{0}=0$, and,for $r \geq 1, f_{r}$ is a linear combination of $2 q$ terms of the form $\xi_{i}-\eta_{i}$, where $\xi_{i}, \eta_{i} \in S_{r-1, s+1}$, and where

$$
\begin{aligned}
A_{n} & =\sum x_{n-1} \cdots x_{1} i_{r} \cdots i_{1} j_{1}^{-1} \cdots j_{s}^{-1} v_{1}^{-1} \cdots v_{n+1}^{-1} \\
B_{n} & =\sum u_{n+1}^{-1} \cdots u_{1}^{-1} l_{s}^{-1} \cdots l_{1}^{-1} k_{1} \cdots k_{r} y_{1} \cdots y_{n-1} \\
C_{n} & =\sum x_{n+1} \cdots x_{1} i_{r} \cdots i_{1} j_{1}^{-1} \cdots j_{s}^{-1} v_{1}^{-1} \cdots v_{n}^{-1} \\
D_{n} & =\sum u_{n}^{-1} \cdots u_{1}^{-1} l_{s}^{-1} \cdots l_{1}^{-1} k_{1} \cdots k_{r} y_{1} \cdots y_{n+1} \\
E_{n} & =\sum x_{1} u_{n}^{-1} \cdots u_{1}^{-1} l_{s}^{-1} \cdots l_{1}^{-1} k_{1} \cdots k_{r} y_{1} \cdots y_{n} \\
F_{n} & =\sum x_{n} \cdots x_{1} i_{r} \cdots i_{1} j_{1}^{-1} \cdots j_{s}^{-1} v_{1}^{-1} \cdots v_{n}^{-1} y_{1}
\end{aligned}
$$

and where again the sums are over the $x_{i}$ 's, $y_{i}$ 's, $u_{i}$ 's and $v_{i}$ 's for which all the terms in the sums are in normal form, as written, except that in $E_{n}$ we sum over $x_{1}$ and over the $u_{i}$ 's and $y_{i}$ 's so that $u_{n}^{-1} \cdots u_{1}^{-1} l_{s}^{-1} \cdots l_{1}^{-1} k_{1} \cdots k_{r} y_{1} \cdots y_{n}$ is in normal form and $x_{1} \neq u_{n}$, and in $F_{n}$, we sum over $y_{1}$ and over the $v_{i}$ 's and $x_{i}$ 's so that $x_{n} \cdots x_{1} i_{r} \cdots i_{1} j_{1}^{-1} \cdots j_{s}^{-1} v_{1}^{-1} \cdots v_{n}^{-1}$ is in normal form and $y_{1} \neq v_{n}$.

PROOF. We first derive this formula when $n=1$. The calculation is then slightly different in the cases $r=0$ and $r \geq 1$, as we shall see. Now

$$
\begin{aligned}
\chi^{+} f_{0}-f_{0} \chi^{+}= & l_{s-1}^{-1} \cdots l_{1}^{-1} k_{1} \cdots k_{r}-i_{r} \cdots i_{1} j_{1}^{-1} \cdots j_{s-1}^{-1} \\
& +\sum x_{1} l_{s}^{-1} \cdots l_{1}^{-1} k_{1} \cdots k_{r}-\sum i_{r} \cdots i_{1} j_{1}^{-1} \cdots j_{s}^{-1} y_{1} \\
= & w^{\prime}-w^{\prime \prime}+E_{0}-F_{0}
\end{aligned}
$$

(where the sums are over $x_{1}$ such that $x_{1} \neq l_{s}$ and over $y_{1}$ such that $y_{1} \neq j_{s}$ ). Next,

$$
f_{1}=\frac{1}{2 q^{2}}\left\{\sum x_{1} i_{r} \cdots i_{1} j_{1}^{-1} \cdots j_{s}^{-1} v_{1}^{-1}+\sum u_{1}^{-1} l_{s}^{-1} \cdots l_{1}^{-1} k_{1} \cdots k_{r} y_{1}\right\}
$$


and so

$$
\begin{aligned}
\chi^{+} f_{1} & -f_{1} \chi^{+} \\
= & \frac{1}{2}\left\{\frac{1}{q} \sum u_{1}^{-1} i_{r} \cdots i_{1} j_{1}^{-1} \cdots j_{s}^{-1} v_{1}^{-1}+\frac{1}{q^{2}} \sum x_{2} x_{1} i_{r} \cdots i_{1} j_{1}^{-1} \cdots j_{s}^{-1} v_{1}^{-1}\right. \\
& +\sum l_{s}^{-1} \cdots l_{1}^{-1} k_{1} \cdots k_{r} y_{1}+\frac{1}{q^{2}} \sum x_{1} u_{1}^{-1} l_{s}^{-1} \cdots l_{1}^{-1} k_{1} \cdots k_{r} y_{1} \\
& -\sum x_{1} i_{r} \cdots i_{1} j_{1}^{-1} \cdots j_{s}^{-1}-\frac{1}{q^{2}} \sum x_{1} i_{r} \cdots i_{1} j_{1}^{-1} \cdots j_{s}^{-1} v_{1}^{-1} y_{1} \\
& \left.-\frac{1}{q} \sum u_{1}^{-1} l_{s}^{-1} \cdots l_{1}^{-1} k_{1} \cdots k_{r} v_{1}^{-1}-\frac{1}{q^{2}} \sum u_{1}^{-1} l_{s}^{-1} \cdots l_{1}^{-1} k_{1} \cdots k_{r} y_{1} y_{2}\right\} \\
= & \frac{1}{2}\left\{\frac{1}{q} P_{0}+\frac{1}{q^{2}} C_{1}+D_{0}+\frac{1}{q^{2}} E_{1}-C_{0}-\frac{1}{q^{2}} F_{1}-\frac{1}{q} Q_{0}-\frac{1}{q^{2}} D_{1}\right\}, \quad \text { say. }
\end{aligned}
$$

Here the ranges of the sums are as explained above. Some comment is needed for the sums $P_{0}$ and $Q_{0}$. Assume that $r \geq 1$. Given $u_{1} \in P$, we have

$$
\sharp\left\{\left(x_{2}, x_{1}\right) \in P^{2}:\left(x_{2}, x_{1}, u_{1}\right) \in \mathscr{T} \text { and } i_{r} \notin \lambda\left(x_{1}\right)\right\}= \begin{cases}q & \text { if } u_{1} \neq i_{r} \\ 0 & \text { if } u_{1}=i_{r} .\end{cases}
$$

So the sum $P_{0}$ is over $u_{1}, v_{1} \in P$ for which $j_{s} \notin \lambda\left(v_{1}\right)$ and $u_{1} \neq i_{r}$. If however $r=0$,

$$
\sharp\left\{\left(x_{2}, x_{1}\right) \in P^{2}:\left(x_{2}, x_{1}, u_{1}\right) \in \mathscr{T} \text { and } x_{1} \neq j_{1}\right\}= \begin{cases}q & \text { if } u_{1} \in \lambda\left(j_{1}\right) \\ q+1 & \text { if } u_{1} \notin \lambda\left(j_{1}\right)\end{cases}
$$

and so $(1 / q) P_{0}$ must be replaced by

$$
\frac{1}{q} \sum_{u_{1} \in \lambda\left(j_{1}\right)} u_{1}^{-1} j_{1}^{-1} \cdots j_{s}^{-1} v_{1}^{-1}+\frac{q+1}{q^{2}} \sum_{u_{1} \notin \lambda\left(j_{1}\right)} u_{1}^{-1} j_{1}^{-1} \cdots j_{s}^{-1} v_{1}^{-1}
$$

the sums also extending over $v_{1}$ such that $j_{s} \notin \lambda\left(v_{1}\right)$. Similarly, the sum in $Q_{0}$ extends over $u_{1}, v_{1} \in P$ for which $u_{1} \notin \lambda\left(l_{s}\right)$ and $v_{1} \neq k_{r}$, but when $r=0$, $-(1 / q) Q_{0}$ must be replaced by

$$
-\frac{1}{q} \sum_{l_{1} \in \lambda\left(v_{1}\right)} u_{1}^{-1} l_{s}^{-1} \cdots l_{1}^{-1} v_{1}^{-1}-\frac{q+1}{q^{2}} \sum_{l_{1} \notin \lambda\left(v_{1}\right)} u_{1}^{-1} l_{s}^{-1} \cdots l_{1}^{-1} v_{1}^{-1}
$$

the sums also extending over $u_{1}$ such that $u_{1} \notin \lambda\left(l_{s}\right)$. Also, when $r=0$,

$$
C_{0}=\sum_{x_{1}: x_{1} \neq j_{1}} x_{1} j_{1}^{-1} \cdots j_{s}^{-1}
$$


so that $C_{0}=E_{0}$ because $j_{1}=l_{s}$. Similarly, $D_{0}=F_{0}$ when $r=0$.

Next,

$$
g_{1}=\frac{-1}{2 q}\left\{\sum i_{r} \cdots i_{1} j_{1}^{-1} \cdots j_{s}^{-1} v_{1}^{-1}+\sum u_{1}^{-1} l_{s}^{-1} \cdots l_{1}^{-1} k_{1} \cdots k_{r}\right\}
$$

and so, when $r \geq 1$,

$$
\begin{aligned}
\chi^{-} g_{1} & -g_{1} \chi^{-} \\
= & \frac{1}{2}\left\{\sum i_{r} \cdots i_{1} j_{1}^{-1} \cdots j_{s}^{-1} y_{1}+\frac{1}{q} \sum i_{r} \cdots i_{1} j_{1}^{-1} \cdots j_{s}^{-1} v_{1}^{-1} v_{2}^{-1}\right. \\
& +\frac{1}{q} \sum u_{1}^{-1} l_{s}^{-1} \cdots l_{1}^{-1} k_{1} \cdots k_{r-1}+\frac{1}{q} \sum u_{1}^{-1} l_{s}^{-1} \cdots l_{1}^{-1} k_{1} \cdots k_{r} v_{1}^{-1} \\
& -\frac{1}{q} \sum i_{r-1} \cdots i_{1} j_{1}^{-1} \cdots j_{s}^{-1} v_{1}^{-1}-\frac{1}{q} \sum u_{1}^{-1} i_{r} \cdots i_{1} j_{1}^{-1} \cdots j_{s}^{-1} v_{1}^{-1} \\
& \left.-\sum x_{1} l_{s}^{-1} \cdots l_{1}^{-1} k_{1} \cdots k_{r}-\frac{1}{q} \sum u_{2}^{-1} u_{1}^{-1} l_{s}^{-1} \cdots l_{1}^{-1} k_{1} \cdots k_{r}\right\} \\
= & \frac{1}{2}\left\{F_{0}+\frac{1}{q} A_{1}+\frac{1}{q} R_{0}+\frac{1}{q} Q_{0}-\frac{1}{q} S_{0}-\frac{1}{q} P_{0}-E_{0}-\frac{1}{q} B_{1}\right\}, \quad \text { say. }
\end{aligned}
$$

When $r=0,(1 / q) R_{0}+(1 / q) Q_{0}$ must be replaced by

$$
\frac{1}{q} \sum_{v_{1}: l_{1} \in \lambda\left(v_{1}\right)} u_{1}^{-1} l_{s}^{-1} \cdots l_{1}^{-1} v_{1}^{-1}+\frac{1}{q} \sum_{v_{1}: l_{1} \notin \lambda\left(v_{1}\right)} u_{1}^{-1} l_{s}^{-1} \cdots l_{1}^{-1} v_{1}^{-1}
$$

the sums also extending over $u_{1} \in P$ such that $u_{1} \notin \lambda\left(l_{s}\right)$. Also, $-(1 / q) S_{0}-$ $(1 / q) P_{0}$ must be replaced by

$$
-\frac{1}{q} \sum_{u_{1} \in \lambda\left(j_{1}\right)} u_{1}^{-1} j_{1}^{-1} \cdots j_{s}^{-1} v_{1}^{-1}-\frac{1}{q} \sum_{u_{1} \notin \lambda\left(j_{1}\right)} u_{1}^{-1} j_{1}^{-1} \cdots j_{s}^{-1} v_{1}^{-1}
$$

the sums also extending over $v_{1} \in P$ such that $j_{s} \notin \lambda\left(v_{1}\right)$.

Suppose that $r=0$. We've noted that $C_{0}=E_{0}$ and $D_{0}=F_{0}$. Also, the first parts of (6.6) and (6.9) cancel, as do the first parts of (6.7) and (6.8), and the second parts of (6.6) and (6.7) cancel, as do the second parts of (6.8) and (6.9). Combining these facts, we find that

$$
\begin{aligned}
\left(\chi^{+}\left(f_{0}+f_{1}\right)-\right. & \left.\left(f_{0}+f_{1}\right) \chi^{+}\right)+\left(\chi^{-} g_{1}-g_{1} \chi^{-}\right) \\
& =w^{\prime}-w^{\prime \prime}+\frac{1}{2 q}\left(A_{1}-B_{1}\right)+\frac{1}{2 q^{2}}\left(C_{1}-D_{1}+E_{1}-F_{1}\right)
\end{aligned}
$$


which is (6.5) for $n=1, r=0$ and $f_{0}=0$.

When $r \geq 1$, we obtain (6.5) for $n=1$ when we set

$$
f_{r}=\frac{1}{2}\left(E_{0}-C_{0}\right)-\frac{1}{2}\left(F_{0}-D_{0}\right)+\frac{1}{2 q}\left(R_{0}-S_{0}\right) .
$$

Using the Lemma 2.1, as re-interpreted after (6.4), we see that

$$
E_{0}-C_{0}=\left\{\sum_{x_{1}: l\left(x_{1} w\right) \geq l(w)} x_{1} w-\sum_{x_{1}: l\left(x_{1} w\right)=l(w)+1} x_{1} w\right\}=\sum_{x_{1}: l\left(x_{1} w\right)=l(w)} x_{1} w
$$

is a sum of $q$ elements of $S_{r-1, s+1}$. Similarly, $F_{0}-D_{0}$ is a sum of $q$ elements of $S_{r-1, s+1}$. Thus $f_{r}$ is a sum of $2 q$ terms $c_{i}\left(\xi_{i}-\eta_{i}\right)$, where $c_{i}=1 / 2$ for $q i$ 's, and $c_{i}=1 / 2 q$ for $q i$ 's.

One now proves (6.5) by induction on $n$, using the formula, valid if $n \geq 1$ for any $r \geq 0$,

$$
\begin{aligned}
& \left(\chi^{+} f_{n+1}-f_{n+1} \chi^{+}\right)+\left(\chi^{-} g_{n+1}-g_{n+1} \chi^{-}\right) \\
& =\frac{1}{2 q^{2 n-1}}\left(B_{n}-A_{n}\right)+\frac{1}{2 q^{2 n}}\left(D_{n}-C_{n}+F_{n}-E_{n}\right) \\
& \quad+\frac{1}{2 q^{2 n+1}}\left(A_{n+1}-B_{n+1}\right)+\frac{1}{2 q^{2 n+2}}\left(C_{n+1}-D_{n+1}+E_{n+1}-F_{n+1}\right)
\end{aligned}
$$

which is derived by calculations similar to those for $\chi^{+} f_{1}-f_{1} \chi^{+}$and $\chi^{-} g_{1}-g_{1} \chi^{-}$.

LEMMA 6.4. Let $r, s \geq 0$, and let $V^{\prime}$ (respectively, $V^{*}$ ) denote the linear span (in the group algebra) of the set of elements of the form $w^{\prime}-w^{\prime \prime}$ (respectively, $w^{*}-w^{* *}$ ), where $w \in S_{r, s+1}$ (respectively, $w \in S_{r+1, s}$ ) (using the notation defined before Lemma 6.3). Let $\xi, \tilde{\xi} \in S_{r, s}$. Then $\xi-\tilde{\xi}$ is in $V^{\prime}+V^{*}$.

PROOF. Write

$$
\xi=i_{r} \cdots i_{1} j_{1}^{-1} \cdots j_{s}^{-1}=l_{s}^{-1} \cdots l_{1}^{-1} k_{1} \cdots k_{r}
$$

and

$$
\tilde{\xi}=\tilde{l}_{r} \cdots \tilde{l}_{1} \tilde{\jmath}_{1}^{-1} \cdots \tilde{\jmath}_{s}^{-1}=\tilde{l}_{s}^{-1} \cdots \tilde{l}_{1}^{-1} \tilde{k}_{1} \cdots \tilde{k}_{r} .
$$

Now the left normal form $l_{s}^{-1} \cdots l_{1}^{-1} k_{1} \cdots k_{r}$ of $\xi$ is obtained from the right normal form by 'passing the $j_{v}^{-1}$ 's from right to left'. After $v$ steps, where $0 \leq v \leq s$, we have

$$
\xi=l_{s}^{-1} \cdots l_{s-\nu+1}^{-1} i_{r}^{(v)} \cdots i_{1}^{(v)} j_{v+1}^{-1} \cdots j_{s}^{-1}
$$


and $i_{r}^{(0)} \cdots i_{1}^{(0)}=i_{r} \cdots i_{1}$ and $i_{r}^{(s)} \cdots i_{1}^{(s)}=k_{1} \cdots k_{r}$. Now let $j_{1}^{\prime-1} \cdots j_{s}^{\prime-1} \in S_{0, s}$ with $j_{s} \notin \lambda\left(j_{1}^{\prime}\right)$. Consider

$$
w=i_{r} \cdots i_{1} j_{1}^{-1} \cdots j_{s}^{-1} j_{1}^{\prime-1} \in S_{r, s+1} .
$$

Then $w=l_{s}^{-1} i_{r}^{(1)} \cdots i_{1}^{(1)} j_{2}^{-1} \cdots j_{s}^{-1} j_{1}^{\prime-1}$, and so

$$
w^{\prime \prime}-w^{\prime}=i_{r} \cdots i_{1} j_{1}^{-1} \cdots j_{s}^{-1}-i_{r}^{(1)} \cdots i_{1}^{(1)} j_{2}^{-1} \cdots j_{s}^{-1} j_{1}^{\prime-1} \in V^{\prime} .
$$

Repeating this, we find that

$i_{r}^{(\nu-1)} \cdots i_{1}^{(\nu-1)} j_{v}^{-1} \cdots j_{s}^{-1} j_{1}^{\prime-1} \cdots j_{\nu-1}^{\prime-1}-i_{r}^{(\nu-1)} \cdots i_{1}^{(\nu-1)} j_{v+1}^{-1} \cdots j_{s}^{-1} j_{1}^{\prime-1} \cdots j_{\nu}^{\prime-1} \in V^{\prime}$

for $v=1, \ldots, s$. Adding, we have

$$
i_{r} \cdots i_{1} j_{1}^{-1} \cdots j_{s}^{-1}-k_{1} \cdots k_{r} j_{1}^{\prime-1} \cdots j_{s}^{\prime-1} \in V^{\prime} .
$$

Now suppose that $\xi, \tilde{\xi} \in S_{r, s}$ are as above, where $s \geq 1$, and that $k_{1} \cdots k_{r}=$ $\tilde{k}_{1} \cdots \tilde{k}_{r}$. Pick $j_{1}^{\prime-1} \cdots j_{s}^{\prime-1} \in S_{0, s}$ with $j_{s}, \tilde{j}_{s} \notin \lambda\left(j_{1}^{\prime}\right)$, and we obtain (6.10) and also

$$
\tilde{l}_{r} \cdots \tilde{l}_{1} \tilde{j}_{1}^{-1} \cdots \tilde{j}_{s}^{-1}-k_{1} \cdots k_{r} j_{1}^{\prime-1} \cdots j_{s}^{\prime-1} \in V^{\prime} .
$$

Subtracting (6.11) from (6.10), we get $\xi-\tilde{\xi} \in V^{\prime}$. This, in particular, proves the lemma when $r=0$.

By an entirely analogous argument, one shows that if $r \geq 1$ and $l_{s}^{-1} \cdots l_{1}^{-1}=$ $\tilde{l}_{s}^{-1} \cdots \tilde{l}_{1}^{-1}$, then $\xi-\tilde{\xi} \in V^{*}$, proving the lemma when $s=0$, in particular. Now if $r, s \geq 1$, pick $k_{1}^{\prime} \cdots k_{r}^{\prime} \in S_{r, 0}$ such that $k_{1} \neq l_{1}, \tilde{l}_{1}$. Then the elements $l_{s}^{-1} \cdots l_{1}^{-1} k_{1} \cdots k_{r}-l_{s}^{-1} \cdots l_{1}^{-1} k_{1}^{\prime} \cdots k_{r}^{\prime}, \quad \tilde{l}_{s}^{-1} \cdots \tilde{l}_{1}^{-1} k_{1}^{\prime} \cdots k_{r}^{\prime}-\tilde{l}_{s}^{-1} \cdots \tilde{l}_{1}^{-1} \tilde{k}_{1} \cdots \tilde{k}_{r}$ and $l_{s}^{-1} \cdots l_{1}^{-1} k_{1}^{\prime} \cdots k_{r}^{\prime}-\tilde{l}_{s}^{-1} \cdots \tilde{l}_{1}^{-1} k_{1}^{\prime} \cdots k_{r}^{\prime}$ are in $V^{*}, V^{*}$ and $V^{\prime}$, respectively, so their sum $\xi-\tilde{\xi}$ is in $V^{\prime}+V^{*}$.

PROOF OF THEOREM 6.1. Let $f \in l^{2}\left(\Gamma_{\mathscr{T}}\right)$ be real valued and satisfy $f * \chi^{+}=$ $\chi^{+} * f$ and $f * \chi^{-}=\chi^{-} * f$. We must show that $f$ is constant on each set $S_{r, s}$. Let $\xi, \tilde{\xi} \in S_{r, s}$. We must show that $\langle\xi-\tilde{\xi}, f\rangle=0$. By the last lemma, we may suppose that $\xi-\tilde{\xi}=w^{\prime}-w^{\prime \prime}$, where $w \in S_{r, s+1}$, or $\xi-\tilde{\xi}=w^{*}-w^{* *}$, where $w \in S_{r+1, s}$. Because $\check{f} * \chi^{+}=\chi^{+} * \check{f}$ and $\check{f} * \chi^{-}=\chi^{-} * \check{f}$, where $\check{f}(x)=f\left(x^{-1}\right)$, and because $\left(w^{-1}\right)^{*}=\left(w^{\prime \prime}\right)^{-1}$ and $\left(w^{-1}\right)^{* *}=\left(w^{\prime}\right)^{-1}$, we may suppose $\xi-\tilde{\xi}=w^{\prime}-w^{\prime \prime}$, where $w \in S_{r, s+1}$. So let $w \in S_{r, s}$, where $s \geq 1$, we show that $f\left(w^{\prime}\right)=f\left(w^{\prime \prime}\right)$. 
If we consider the inner product of $f$ with both sides of (6.5), we see, using $\langle h, g\rangle=h * \breve{f}(e)=\breve{f} * h(e), \breve{f} * \chi^{+}=\chi^{+} * \breve{f}$ and $\breve{f} * \chi^{-}=\chi^{-} * \check{f}$, that $0=f\left(w^{\prime}\right)-f\left(w^{\prime \prime}\right)+\left\langle f_{r}, f\right\rangle+\left\langle\frac{1}{2 q^{2 n-1}}\left(A_{n}-B_{n}\right)+\frac{1}{2 q^{2 n}}\left(C_{n}-D_{n}+E_{n}-F_{n}\right), f\right\rangle$

Now $A_{n}$, regarded as a function on $\Gamma_{\mathscr{T}}$, is the characteristic function of a set of $q^{4 n}$ distinct elements in $S_{r+n-1, s+n+1}\left(\left(q^{2}+q\right) q^{2(2 n-1)}\right.$ elements when $\left.r=0\right)$. Thus $\left\|A_{n}\right\|_{2} \leq \sqrt{q^{2}+q} q^{2 n-1}$, and so

$$
\left|\left\langle\frac{1}{q^{2 n-1}} A_{n}, f\right\rangle\right|^{2} \leq\left(q^{2}+q\right) \sum_{x \in S_{r+n-1 . s+n+1}}|f(x)|^{2} \quad \rightarrow 0 \quad \text { as } n \rightarrow \infty
$$

Similarly, the terms involving $B_{n}, C_{n}$ and $D_{n}$ tend to 0 . The terms in $E_{n}$ and $F_{n}$ are slightly different. We can write $E_{n}=\sum_{x_{1} \in P} x_{1} E_{x_{1}, n}$, where

$$
E_{x_{1}, n}=\sum u_{n}^{-1} \cdots u_{1}^{-1} l_{s}^{-1} \cdots l_{1}^{-1} k_{1} \cdots k_{r} y_{1} \cdots y_{n}
$$

the sum being over the $u_{i}$ 's and $y_{i}$ 's so that the terms are in normal form as written and $u_{n} \neq x_{1}$. Then

$$
\left\|E_{n}\right\|_{2} \leq \sum_{x_{1} \in P}\left\|E_{x_{1}, n}\right\|_{2} \leq\left(q^{2}+q+1\right) \sqrt{q^{2}+q} q^{2 n-1}
$$

Because $E_{n}$ is a linear combination of terms in $S_{r+n+1, s+n} \cup S_{r+n-1, s+n+1}$, $\left|\left\langle\frac{1}{q^{2 n}} E_{n}, f\right\rangle\right| \rightarrow 0$ as $n \rightarrow \infty$. We can similarly deal with the term involving $F_{n}$.

We thus obtain $0=f\left(w^{\prime}\right)-f\left(w^{\prime \prime}\right)+\left\langle f_{r}, f\right\rangle$ When $r=0, f_{0}=0$ and we have $f\left(w^{\prime}\right)=f\left(w^{\prime \prime}\right)$, so that we have proved that for any $s \geq 0$, if $\xi_{1}, \xi_{2} \in S_{0, s}$, then $f\left(\xi_{1}\right)=f\left(\xi_{2}\right)$. Assume that $r \geq 1$ and that it has been proved that for any $n \geq 0$ and for any $\xi_{1}, \xi_{2} \in S_{r-1, n}, f\left(\xi_{1}\right)=f\left(\xi_{2}\right)$ holds. Then $\left\langle f_{r}, f\right\rangle=0$ because of the form of $f_{r}$. This completes the proof.

\section{References}

[1] K. S. Brown, Buildings (Springer, New York, 1989).

[2] D. I. Cartwright, A. M. Mantero, T. Steger and A. Zappa, 'Groups acting simply transitively on the vertices of a building of type $\tilde{A}_{2}$ I', Geom. Ded. 47 (1993), 143-166.

[3] — - 'Groups acting simply transitively on the vertices of a building of type $\tilde{A}_{2}$ II: the cases $q=2$ and $q=3^{\prime}$, Geom. Ded. 47 (1993), 167-223. 
[4] M. G. Cowling and T. Steger, 'The irreducibility of restrictions of unitary representations to lattices', J. Reine Angew. Math. 420 (1991), 85-98.

[5] A. Figà-Talamanca and C. Nebbia, Harmonic analysis and representation theory for groups acting on homogeneous trees, London Math. Soc. Lecture Note Ser. 162 (Cambridge University Press, Cambridge, 1991).

[6] A. Figà-Talamanca and M. A. Picardello, Harmonic analysis on free groups, Lect. Notes Pure Appl. Math. 87 (Marcel Dekker, New York, 1983).

[7] I. G. Macdonald, Spherical functions on a group of p-adic type, Ramanujan Inst. Publications 2 (University of Madras, 1971).

[8] G. K. Pedersen, Analysis now, Graduate Texts in Math. 118 (Springer, New York, 1989).

[9] T. Pytlik, 'Radial functions on free groups and a decomposition of the regular representation into irreducible components', J. Reine Angew. Math. 326 (1981), 124-135.

[10] M. Ronan, Lectures on Buildings, Perspect. in Math. 7 (Academic Press, New York, 1989).

[11] J-P. Serre, Trees (Springer-Verlag, New York, 1980).

[12] J. Tits, 'Spheres of radius 2 in triangle buildings. I', in: Finite geometries, buildings, and related topics (eds. W. Kantor et al.) (Clarendon Press, Oxford, 1990) pp. 17-28.

School of Mathematics and Statistics

The University of Sydney

NSW 2006

Australia
Institute of Mathematics The University of Wrocław

pl. Grunwaldzki $2 / 4$ 50-384 Wrocław

Poland 